\title{
Horsemint as a potential raw material for the food industry: survey on the chemistry of a less studied mint species
}

\author{
Katalin Patonay (1) - Éva Németh-Zámboriné
}
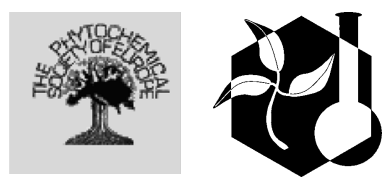

Received: 13 February 2020/ Accepted: 25 August 2020/Published online: 7 September 2020

(C) The Author(s) 2020

\begin{abstract}
Horsemint (Mentha longifolia L), is wildgrowing species, widespread in Eurasia and Africa. The review focuses on its potential utilization as a preservative and flavoring in the food industry based on the polyphenolic and terpenoid composition. Several phenolic antioxidants were detected in horsemint, among which rosmarinic acid may have a key role. Nineteen other acids, and fifty-five flavonoids (six which are de novo) were also identified. The antiradical efficacy in horsemint extract has not yet been adequately justified. Similarly, systematic screening of the flavonoid composition of the species is lacking. Horsemint essential oils possesses an outstandingly wide variability in composition which may serve as basis of special flavoring or antimicrobial agents. The efficacy of horsemint volatiles have been demonstrated against more than twenty microbes. As current literature of horsemint lacks comparable results, the present review provides the broadest and therefore, a critical overview, on its most important
\end{abstract}

K. Patonay ( $\square)$

Food and Wine Research Institute, Eszterházy Károly

University, 6G Leányka st., Eger 3300, Hungary

e-mail: patonay.katalin@uni-eszterhazy.hu

É. Németh-Zámboriné

Department of Medicinal and Aromatic Plants, Szent István University, 29-43 Villányi st., Budapest 1118, Hungary

e-mail: zamborine.nemeth.eva@kertk.szie.hu secondary compounds and the factors influencing their accumulation.

Keywords Mentha longifolia · Polyphenols · Antioxidants · Volatiles · Antimicrobial · Food
Abbreviations
AAPH 2,2'-Azobis(2-amidinopropane) dihydrochloride
AO Antioxidant
DCM Dichloromethane
dp Dry plant material
DPPH 2,2'-Diphenyl pycrylhydrazyl
$\mathrm{EC}_{50}$ Effective concentration-50
EO Essential oil
FRAP Ferrous reducing activity
IC\% Inhibitory concentration in percentage
MIC Minimal inhibitory concentration
RA Rosmarinic acid
TAC Total antioxidant capacity
TF Total flavonoid content
THD Total hydroxycinnamic acid content

\section{Introduction}

Mentha longifolia $\mathrm{L}$, horsemint, wild or biblical mint is a perennial herb belonging to the Mentha genus in the Nepetoideae subfamily of Lamiaceae. According 
Table 1 Subspecies of Mentha longifolia $\mathrm{L}$ and the regions where they were recorded, based on Tucker and Naczi 2007

\begin{tabular}{ll}
\hline Subspecies & Country or region \\
\hline ssp. calliantha & Southwestern Iran, Eastern Anatolia \\
ssp. capensis & Cape Colony, Zimbabwe, Lesotho, Namibia \\
ssp. caucasica & Caucasus \\
ssp. cyprica & Cyprus, mountainous regions \\
ssp. diabolina & Eastern Europe; Asia \\
ssp. dumortieri & Belgium \\
ssp. erminea & Crete, Southern and Eastern Greece, Turkey \\
ssp. grisella & Asia Minor, and Greece, Macedonia, Romania, Hungary. \\
ssp. hymalaiensis & Himalaya. Afghanistan \\
ssp. lavandulaceae & Spain \\
ssp. longifolia & Whole Europe \\
ssp. minutiflora & Hungary, Macedonia and Crete \\
ssp. modesta & Asia Minor, Iran, Tibet \\
ssp. mollis & Romania, Former Yugoslavia \\
ssp.noëana & Southeastern Anatolia, Western Iran, Iraq. \\
ssp. pellita & Syria and Ethiopia \\
ssp. polyadena & South Africa; Lesotho \\
ssp. royleana & Siberia, Asia Minor, Iran, Afghanistan, Tibet \\
ssp. schimperi & Ethiopia, Yemen, Sinai peninsula \\
ssp. syriaca & Syria and Ethiopia \\
ssp. typhioides & Aegean region, Northern Iran, Northern Iraq, Egypt, Lebanon and Israel \\
ssp. wissii & South Africa, Namibia \\
\hline &
\end{tabular}

to the monography of the genus (Tucker and Naczi 2007), its natural distribution area is the largest among wild-growing Mentha species, covering temperate and mediterranean regions of Eurasia and Africa. (Tucker and Naczi 2007; Sevindik et al. 2017; Sevindik 2018).

This may be evaluated as a sign of adaptivity. The large number of taxa included by the species indicates its genetic diversity. The monography lists 22 subspecies of $M$. longifolia described from different regions of the world (Table 1).

In Turkey, Iraq, Iran, Pakistan and Arabic countries, leaves or flowering shoots are used as a spice, i.e. for dairy specialties (Tunçturk et al. 2011; Mahmoudi et al. 2012; Ehsani and Mahmoudi 2012), as leafy vegetables, herbal tea and an ethnomedicinal remedy (Ghoulami et al. 2001; Başer et al. 2012; Iqbal et al. 2013; Mikaili et al. 2013; Murad et al. 2016; Sevindik et al. 2017). A recent review of Farzaei et al. (2017) provides ethnopharmacological data in the aforementioned regions, with a wide variety of traditional indications. Beside collection, $M$. longifolia is reported to be cultivated in Tunisia (Hajlaoui et al. 2009) and its intraspecific taxon M. longifolia var. schimperii syn. ssp. schimperii in Sudan (Younis and Beshir 2011).

In Europe, M. longifolia is far less known and used, contrary to its abundance in wet meadows, forests and ruderal areas. A couple of works have however, been published on analyzing constituents and/or preparations of $M$. longifolia due to potential industry-related uses (Dudai et al. 2006; Güllüce et al. 2007; Krzyzanowska et al. 2011; Bertoli et al. 2011; Orhan et al. 2012). Beside them, some works are also available on $M$. longifolia as a medicinal plant, primarily of antiinflammatory and chemoprotective effects (Mimica-Dukić et al. 1996, 1999; Shen et al. 2011; Baris et al. 2011; Vladimir-Knežević et al. 2014). Nevertheless, M. longifolia is less studied as other Mentha species, partially concerning its nonvolatile constituents. Until now it has not been used either industrially or pharmaceutically on a largescale. However, it seems to be a cheap and prosperous 
additive in numerous products. A promising application may be the usage of $M$. longifolia polyphenols in the food industry as antioxidants (AOs) to increase shelf life. This potential use may be considered with regard to the high demand of plant-originated antioxidants (AOs) and in parallel, health concerns due to some synthetic phenolic AOs (Shahidi and Ambigaipalan 2015). Another alternative may be utilization of the volatiles of selected $M$. longifolia chemotypes against foodborne microbes, or as flavoring agents. In the present review, these two potential ways of utilization of this adaptive species, having a large tolerance for various habitats, in different preparations are in focus. Therefore, a detailed survey was carried out on the respective secondary compounds and a thorough evaluation is presented.

\section{Materials and search strategy}

Beside the comprehensive review Labiatae flavonoids and their bioactivity (Ulubelen et al. 2005) as a starting point, studies on M. longifolia and related species evaluated here were primarily obtained from electronic databases, namely SpringerLink, ScienceDirect, Journal of Agricultural and Food Chemistry, JEOR, Wiley Online Library, Taylor and Francis and MDPI. PubMed, Google Scholar and ResearchGate were used to search reliable but less known sources like the study of Jahan et al. (2001) on a novel flavone detected from ML. To check the background of journals providing some of the latter, Scimago was used. References in available publications were also screened in further sources like dissertations or less cited articles. One example was the study on phenolics of M. x piperita (Guédon and Pasquier 1994) referring to the earliest available work on $M$. longifolia flavonoids (Bourwieg and Pohl 1973) as a nowadays less known source to exploit. Further references considered to be necessary (e.g. studies dealing with structure-AO activity relationships of flavonoids), were searched also at the above mentioned databases. In the present review the cited data on concentrations of phenolics in M. longifolia will be given both in the original measuring units as they were published and also, in the majority of cases, in $\mathrm{mg} / \mathrm{kg}$ dry plant material $(\mathrm{mg} / \mathrm{kg} \mathrm{dp})$ for the sake of better comparability.

\section{Cinnamic acid derivatives in ML}

Rosmarinic acid

Rosmarinic acid or 'Labiatae tannin' (further: RA) is the caffeoyl ester of caffeic acid and $3^{\prime}, 4^{\prime}$-dihydroxyphenyllactic acid. Accumulation of RA is characteristic in the Nepetoideae subfamily of the Lamiaceae family. Petersen and Simmonds (2003) summarize RA as adstringent, AO, antiviral, antimutagen and antiinflammatory agent. Investigations on $M$. longifolia phenolics have predominantly been focusing on RA as a potent AO (Dudai et al. 2006; Fialová et al. 2008; Krzyzanowska et al. 2011; Patonay et al. 2017) antiinflammatory (Shen et al. 2011) and anticholinesterase (Vladimir-Knežević et al. 2014) molecule. The available quantitative data dealing with RA content of M. longifolia is summarized in Table 2. However, as large differences are observable in the investigated drug types and plant developmental stages (if defined), comparison of data can not be totally adequate. The most thorough publication on RA and caffeic acid content of $M$. longifolia is the work of Dudai et al. (2006) being the only one analyzing large sample numbers of $M$. longifolia for any phenolics. Results represent the highest RA content available in the literature of $M$. longifolia, covering 20-80 mg/g dp. (20,000-80,000 mg/kg dp). On the other hand, a recent work (Park et al. 2019) gives unconventionally low RA concentration (18.68 $\mu \mathrm{g} / \mathrm{g}$ dp. viz. $18.68 \mathrm{mg} / \mathrm{kg} \mathrm{dp})$ from a single M. longifolia sample of undefined phenophase. In general, there is a relatively large variability in RA concentrations of $M$. longifolia mentioned by different authors, and they seem to be determined not only genetically but might be the result of differences in the plant developmental stage, harvest time (Fialova et al. 2008), cultivation technics, drug types (Krzyzanowska et al. 2011), extraction methods or other factors (Table 2).

Other phenolic acids, esters and phenylpropanoid volatiles

Beside RA, further phenolics have been detected in $M$. longifolia samples. Table 3 summarizes their concentrations. Nepetoidin A and B are reported to be present in M. longifolia (Grayer et al. 2003) as a chemotaxonomical marker of Nepetoideae plants. Salvianolic 
Table 2 Rosmarinic acid content obtained from different Mentha longifolia samples

\begin{tabular}{|c|c|c|c|c|}
\hline \multirow[t]{2}{*}{ References } & \multicolumn{2}{|l|}{ Quantitative data } & \multirow[t]{2}{*}{ Plant part investigated } & \multirow[t]{2}{*}{ Phenophase } \\
\hline & $\begin{array}{l}\text { Concentration } \\
\text { measured }\end{array}$ & $\begin{array}{l}\text { Concentration in } \mathrm{mg} / \mathrm{kg} \\
\mathrm{dp} \text {. }\end{array}$ & & \\
\hline Dudai et al. (2006) & $20-80 \mathrm{mg} / \mathrm{g} \mathrm{dp}$ & $20,000-80,000$ & Leaves and stem & Not defined \\
\hline Fialová et al. (2008) & $1.19 \mathrm{~m} / \mathrm{m} \%$ in $\mathrm{dp}$ & 11,900 & Not defined & Not defined ${ }^{c}$ \\
\hline Fialová et al. (2008) & $0.88 \mathrm{~m} / \mathrm{m} \%$ in $\mathrm{dp}$ & 8800 & Not defined & Not defined \\
\hline Krzyzanowska et al. (2011) & $1.933 \mathrm{mg} / \mathrm{g} \mathrm{dp}$ & 1933 & Not defined & Not defined \\
\hline Krzyzanowska et al. (2011) & $4.445 \mathrm{mg} / \mathrm{g} \mathrm{dp}$ & 4455 & Not defined (in vitro plant) & Not defined \\
\hline Krzyzanowska et al. (2011) & $12.765 \mathrm{mg} / \mathrm{g} \mathrm{dp}$ & 12,765 & Cell suspension & $(-)$ \\
\hline Krzyzanowska et al. (2011) & $21.576 \mathrm{mg} / \mathrm{g} \mathrm{dp}$ & 21,576 & Callus culture & $(-)$ \\
\hline Tahira et al. (2011) & $61.47 \mathrm{mg} / 100 \mathrm{~g}$ & 614.7 & Leaves & $\begin{array}{l}\text { Spring } \\
\text { vegetative }\end{array}$ \\
\hline Shen et al. (2011) & $6.99 \mathrm{mg} / \mathrm{g} \mathrm{dp}$ & 6990 & Aerial p. without flowers & Flowering \\
\hline Shekarchi et al. (2012) & $26.6 \mathrm{mg} / \mathrm{g} \mathrm{dp}$ & 26,600 & Aerial parts & Flowering \\
\hline $\begin{array}{l}\text { Vladimir-Kneževic et al. } \\
\text { (2014) }\end{array}$ & $22.33 \mathrm{mg} / \mathrm{g}$ extract & 1639 & Aerial parts & Flowering \\
\hline $\begin{array}{l}\text { Elansary and Mahmoud } \\
(2015)\end{array}$ & $40.91 \mathrm{mg} / \mathrm{g}$ extract & $\mathrm{NA}^{\mathrm{a}}$ & Leaves & Not defined \\
\hline Bahadori et al. (2018) & $2225-6260 \mu \mathrm{g} / \mathrm{g}$ & $\mathrm{NA}^{\mathrm{a}}$ & $\begin{array}{l}\text { Leaves, flowers, juvenile } \\
\text { stems }\end{array}$ & Flowering \\
\hline Patonay et al. (2017) & $6418-11,366 \mathrm{mg} / \mathrm{kg}$ & $6418-11,366^{\mathrm{b}}$ & Leaves, stem, inflorescence & Flowering \\
\hline Park et al. (2019) & $18.68 \mathrm{ug} / \mathrm{g} \mathrm{dp}$ & 18.68 & Leaves and stem & Not defined ${ }^{\mathrm{e}}$ \\
\hline
\end{tabular}

${ }^{\mathrm{a}}$ Concentration given in extracts without providing yields, thus in $\mathrm{mg} / \mathrm{kg} \mathrm{dp}$ is not possible to calculate

${ }^{\mathrm{b}}$ Concentration depending on the extraction solvent

${ }^{\mathrm{c}}$ Harvested in July

${ }^{\mathrm{d}}$ Harvested in September

${ }^{\mathrm{e}}$ Harvested in June

acid $\mathrm{L}$ and dedihydro-salvianolic acid was detected by Krzyzanowska and co-workers (2011). It may be important to note that $\mathrm{m} / \mathrm{z}$ data and UV maxima $(283.3,344.4 \mathrm{~nm})$ of dedihydro-salvianolic acid were provided in this study, but molecular structure of a compound with this name was found neither in PubChem, PhenolExplorer, Human Metabolome Database or NIST Webbook, nor in literature. Hexacosyl ferulate and bis-2-ethylhexil-benzene 1,2-dicarboxylate were reported from Mentha longifolia L ssp. noëana sampled in Turkey (Ertaş et al. 2015). This is the first report of them from $M$. longifolia, thus, based on this single reference it is impossible to evaluate the frequency and level of their concentration in horsemint. In general, data of minor phenolic acids in this species outline a rather wide variablity, but unfortunately there is hardly any data about the influencing factors of the accumulation of them until now.

\section{Flavonoids in horsemint}

Table 4 summarizes structural information of flavonoids reported in Mentha longifolia.

\section{Flavanones}

Flavanones ('citrus flavonoids') are usually a dominant or a major flavonoid subclass in mints, together with flavones (Pereira and Cardoso 2013). Regarding M. longifolia, data about them is sporadic and a majority of the references report only the presence of these compounds (eriocitrin, hesperidin and narirutin) without quantitative data. The spectrum of flavanones include relatively widespread compounds (Table 4), but a special flavanone, 4'-methoxy-naringenin-7-Ofucopyranosil-1 $\rightarrow$ 6-glucoside or longitin, reported 
Table 3 Concentration of nonflavonoid phenolics (beyond rosmarinic acid) in Mentha longifolia

\begin{tabular}{|c|c|c|c|c|c|c|}
\hline \multirow[t]{2}{*}{ Subclass } & \multirow[t]{2}{*}{ Name } & \multirow[t]{2}{*}{ References } & \multicolumn{2}{|c|}{ Quantitative data } & \multirow{2}{*}{$\begin{array}{l}\text { Plant part } \\
\text { investigated }\end{array}$} & \multirow{2}{*}{$\begin{array}{l}\text { Plant } \\
\text { phenophase }\end{array}$} \\
\hline & & & $\begin{array}{l}\text { Concentration } \\
\text { measured }\end{array}$ & $\begin{array}{l}\text { Concentration, } \\
\mathrm{mg} / \mathrm{kg} \mathrm{dp} \text {. }\end{array}$ & & \\
\hline \multirow[t]{23}{*}{$\begin{array}{l}\text { Cinnamic acid } \\
\text { derivative }\end{array}$} & trans-Cinnamic acid & Park et al. (2019) & $6.5 \mathrm{ug} / \mathrm{g} \mathrm{dp}$ & 6.5 & Leaves and stem & $\begin{array}{l}\text { Not } \\
\text { defined }^{c}\end{array}$ \\
\hline & trans-Cinnamic acid & $\begin{array}{l}\text { Bahadori et al. } \\
\text { (2018) }\end{array}$ & $\begin{array}{l}\text { 15-79 ug/g } \\
\text { dry extract }\end{array}$ & $\mathrm{NA}^{\mathrm{a}}$ & $\begin{array}{c}\text { Flowers, leaves, } \\
\text { juvenile stems }\end{array}$ & Flowering \\
\hline & $\begin{array}{l}\text { para-Coumaric acid } \\
\text { (bound) }\end{array}$ & $\begin{array}{l}\text { Dudai et al. } \\
\text { (2006) }\end{array}$ & $\begin{array}{l}\text { ca. } \\
25-250 \mathrm{mg} / \\
\text { g dp }\end{array}$ & ca. $250-2500$ & Leaves and stem & Not defined \\
\hline & para-Coumaric acid & $\begin{array}{l}\text { Benedec et al. } \\
\text { (2013) }\end{array}$ & $2.687 \mathrm{mg} / \mathrm{dp}$ & 26.9 & Aerial parts & Flowering \\
\hline & para-Coumaric acid & $\begin{array}{l}\text { Bahadori et al. } \\
\text { (2018) }\end{array}$ & $\begin{array}{l}5-15 \mathrm{ug} / \mathrm{g} \text { dry } \\
\text { extract }^{\mathrm{b}}\end{array}$ & $\mathrm{NA}^{\mathrm{a}}$ & $\begin{array}{c}\text { Flowers, leaves, } \\
\text { juvenile stems }\end{array}$ & Flowering \\
\hline & para-Coumaric acid & Park et al. (2019) & $6.15 \mathrm{ug} / \mathrm{g} \mathrm{dp}$ & 6.15 & Leaves and stem & $\begin{array}{l}\text { Not } \\
\text { defined }^{\mathrm{c}}\end{array}$ \\
\hline & ortho-Coumaric acid & $\begin{array}{l}\text { Bahadori et al. } \\
\text { (2018) }\end{array}$ & $\begin{array}{l}\text { 134-328 ug/g } \\
\text { extract }^{\mathrm{b}}\end{array}$ & $\mathrm{NA}^{\mathrm{a}}$ & $\begin{array}{l}\text { Flowers, leaves, } \\
\text { juvenile stems }\end{array}$ & Flowering \\
\hline & Caffeic acid (free) & $\begin{array}{l}\text { Dudai et al. } \\
\text { (2006) }\end{array}$ & $\begin{array}{l}\text { ca. } 10-24 \mathrm{mg} / \\
\text { g dp }\end{array}$ & $10,000-24,000$ & Leaves and stem & Not defined \\
\hline & Caffeic acid (bound) & $\begin{array}{l}\text { Dudai et al. } \\
\text { (2006) }\end{array}$ & $\begin{array}{l}\text { ca. } 6-16 \mathrm{mg} / \mathrm{g} \\
\mathrm{dp}\end{array}$ & $\begin{array}{l}\text { ca. } \\
\quad 6000-16,000\end{array}$ & Leaves and stem & Not defined \\
\hline & Caffeic acid & $\begin{array}{l}\text { Tahira et al. } \\
\text { (2011) }\end{array}$ & $\begin{array}{l}314.8 \mathrm{mg} / \\
100 \mathrm{~g} \mathrm{dp}\end{array}$ & 3418 & Leaves & $\begin{array}{l}\text { Spring } \\
\text { vegetative }\end{array}$ \\
\hline & Caffeic acid & $\begin{array}{l}\text { Benedec et al. } \\
\text { (2013) }\end{array}$ & $<0.2 \mathrm{mg} / \mathrm{dp}$ & $<2.0$ & Aerial parts & Flowering \\
\hline & Caffeic acid & $\begin{array}{l}\text { Vladimír- } \\
\text { Kňezević } \\
\text { (2014) }\end{array}$ & $\begin{array}{l}1.18 \mathrm{mg} / \mathrm{g} \text { dry } \\
\text { extract }\end{array}$ & 86.7 & Aerial parts & Flowering \\
\hline & Caffeic acid & $\begin{array}{l}\text { Patonay et al. } \\
\text { (2017) }\end{array}$ & $\begin{array}{l}\text { 0-273.3 mg/ } \\
\mathrm{kg} \mathrm{dp}\end{array}$ & $0-273.3$ & $\begin{array}{l}\text { Leaves, stem, } \\
\text { inflorescence }\end{array}$ & Flowering \\
\hline & Caffeic acid & $\begin{array}{l}\text { Bahadori et al. } \\
\text { (2018) }\end{array}$ & $\begin{array}{l}\text { 86-119 ug/g } \\
\text { extract }^{\mathrm{b}}\end{array}$ & $\mathrm{NA}^{\mathrm{a}}$ & $\begin{array}{l}\text { Flowers, leaves, } \\
\text { juvenile stems }\end{array}$ & Flowering \\
\hline & Caffeic acid & Park et al. (2019) & $58.50 \mathrm{ug} / \mathrm{g} \mathrm{dp}$ & 58.5 & Leaves and stem & $\begin{array}{l}\text { Not } \\
\text { defined }^{\mathrm{c}}\end{array}$ \\
\hline & Ferulic acid & $\begin{array}{l}\text { Tahira et al. } \\
\text { (2011) }\end{array}$ & $\begin{array}{l}0.94 \mathrm{mg} / 100 \mathrm{~g} \\
\mathrm{dp}\end{array}$ & 9.4 & Leaves & $\begin{array}{l}\text { Spring } \\
\text { vegetative }\end{array}$ \\
\hline & Ferulic acid & $\begin{array}{l}\text { Benedec et al. } \\
\text { (2013) }\end{array}$ & $<0.2 \mathrm{mg} / \mathrm{dp}$ & $<2$ & Aerial parts & Flowering \\
\hline & Ferulic acid & $\begin{array}{l}\text { Patonay et al. } \\
\text { (2017) }\end{array}$ & $\begin{array}{l}0-30.59 \mathrm{mg} / \\
\mathrm{kg} \mathrm{dp}\end{array}$ & ND-30.6 & $\begin{array}{l}\text { Leaves, stem, } \\
\text { inflorescence }\end{array}$ & Flowering \\
\hline & Ferulic acid & Park et al. (2019) & $\begin{array}{l}38.70 \mathrm{ug} / \mathrm{g} \text { dry } \\
\text { extract }\end{array}$ & 38.7 & Leaves and stem & $\begin{array}{l}\text { Not } \\
\text { defined }^{c}\end{array}$ \\
\hline & Hexacosyl ferulate & Ertaş et al. (2015) & $\begin{array}{l}6 \mathrm{mg} / 1350 \mathrm{~g} \\
\mathrm{dp}\end{array}$ & 4.4 & Aerial parts & Not defined \\
\hline & Sinapic acid & $\begin{array}{l}\text { Bahadori et al. } \\
\text { (2018) }\end{array}$ & $\begin{array}{l}\text { 4604-7132 ug/ } \\
\text { g extract }^{\mathrm{b}}\end{array}$ & $\mathrm{NA}^{\mathrm{a}}$ & $\begin{array}{l}\text { Flowers, leaves, } \\
\text { juvenile stems }\end{array}$ & Flowering \\
\hline & Caftaric acid & $\begin{array}{l}\text { Benedec et al. } \\
\text { (2013) }\end{array}$ & $\begin{array}{r}<0.2 \mathrm{mg} / \\
100 \mathrm{~g} \mathrm{dp}\end{array}$ & $<2$ & Aerial parts & Flowering \\
\hline & Chlorogenic acid & $\begin{array}{l}\text { Benedec et al. } \\
\text { (2013) }\end{array}$ & $\begin{array}{r}<0.2 \mathrm{mg} / \\
100 \mathrm{~g} \mathrm{dp}\end{array}$ & $<2$ & Aerial parts & Flowering \\
\hline
\end{tabular}


Table 3 continued

\begin{tabular}{|c|c|c|c|c|c|c|}
\hline \multirow[t]{2}{*}{ Subclass } & \multirow[t]{2}{*}{ Name } & \multirow[t]{2}{*}{ References } & \multicolumn{2}{|c|}{ Quantitative data } & \multirow{2}{*}{$\begin{array}{l}\text { Plant part } \\
\text { investigated }\end{array}$} & \multirow{2}{*}{$\begin{array}{l}\text { Plant } \\
\text { phenophase }\end{array}$} \\
\hline & & & $\begin{array}{l}\text { Concentration } \\
\text { measured }\end{array}$ & $\begin{array}{l}\text { Concentration, } \\
\mathrm{mg} / \mathrm{kg} \text { dp. }\end{array}$ & & \\
\hline & Chlorogenic acid & $\begin{array}{l}\text { Vladimír- } \\
\text { Kňezević et al. } \\
\text { (2014) }\end{array}$ & $\begin{array}{l}1.50 \mathrm{mg} / \mathrm{g} \text { dry } \\
\text { extract }\end{array}$ & 110.1 & Aerial parts & Flowering \\
\hline & Chlorogenic acid & $\begin{array}{l}\text { Bahadori et al. } \\
2018\end{array}$ & $\begin{array}{c}27-64 \mathrm{ug} / \mathrm{g} \\
\text { extract }^{\mathrm{b}}\end{array}$ & $\mathrm{NA}^{\mathrm{a}}$ & $\begin{array}{c}\text { Flowers, leaves, } \\
\text { juvenile stems }\end{array}$ & Flowering \\
\hline & Chlorogenic acid & Park et al. (2019) & $170.90 \mathrm{ug} / \mathrm{g}$ & 170.9 & Leaves and stem & $\begin{array}{l}\text { Not } \\
\text { defined }^{\mathrm{c}}\end{array}$ \\
\hline & Salvianolic acid L & $\begin{array}{c}\text { Krzyzanowska } \\
\text { et al. (2011) }\end{array}$ & $0.285 \mathrm{mg} / \mathrm{g} \mathrm{dp}$ & 285.0 & Not defined & Not defined \\
\hline & $\begin{array}{l}\text { Dedihydro-salvianolic } \\
\text { acid }\end{array}$ & $\begin{array}{l}\text { Krzyzanowska } \\
\text { et al. (2011) }\end{array}$ & $0.084 \mathrm{mg} / \mathrm{g} \mathrm{dp}$ & 84.0 & Not defined & Not defined \\
\hline & Nepetoidin A & $\begin{array}{l}\text { Grayer et al. } \\
\text { (2003) }\end{array}$ & $\begin{array}{l}\text { Quantity not } \\
\text { given }\end{array}$ & NA & Leaves & Flowering \\
\hline & Nepetoidin B & $\begin{array}{l}\text { Grayer et al. } \\
(2003)\end{array}$ & $\begin{array}{l}\text { Quantity not } \\
\text { given }\end{array}$ & NA & Leaves & Flowering \\
\hline \multirow{11}{*}{$\begin{array}{l}\text { Benzoic acid } \\
\text { derivative/ } \\
\text { other }\end{array}$} & Benzoic acid & Park et al. (2019) & $25.84 \mathrm{ug} / \mathrm{g} \mathrm{dp}$ & 25.8 & Leaves and stem & $\begin{array}{l}\text { Not } \\
\text { defined }^{c}\end{array}$ \\
\hline & Vanillic acid & $\begin{array}{l}\text { Patonay et al. } \\
(2017)\end{array}$ & $\begin{array}{l}0-62.17 \mathrm{mg} / \\
\mathrm{kg} \mathrm{dp}\end{array}$ & ND-62.17 & $\begin{array}{l}\text { Leaves, stem, } \\
\text { inflorescence }\end{array}$ & Flowering \\
\hline & Gallic acid & $\begin{array}{l}\text { Patonay et al. } \\
\text { (2017) }\end{array}$ & $\begin{array}{l}0-2583 \mathrm{mg} / \mathrm{kg} \\
\mathrm{dp}\end{array}$ & ND-2583 & $\begin{array}{l}\text { Leaves, stem, } \\
\text { inflorescence }\end{array}$ & Flowering \\
\hline & Gallic acid & $\begin{array}{l}\text { Bahadori et al. } \\
\text { (2018) }\end{array}$ & $\begin{array}{l}2-72 \mathrm{ug} / \mathrm{g} \\
\text { extract }^{\mathrm{b}}\end{array}$ & $\mathrm{NA}^{\mathrm{a}}$ & $\begin{array}{c}\text { Flowers, leaves, } \\
\text { juvenile stems }\end{array}$ & Flowering \\
\hline & Syringic acid & $\begin{array}{l}\text { Patonay et al. } \\
\text { (2017) }\end{array}$ & $\begin{array}{l}0-56.75 \mathrm{mg} / \\
\mathrm{kg} \mathrm{dp}\end{array}$ & ND-56.75 & $\begin{array}{l}\text { Leaves, stem, } \\
\text { inflorescence }\end{array}$ & Flowering \\
\hline & Syringic acid & $\begin{array}{l}\text { Bahadori et al. } \\
\text { (2018) }\end{array}$ & $\begin{array}{l}9-33 \mathrm{ug} / \mathrm{g} \\
\text { extract }^{\mathrm{b}}\end{array}$ & $\mathrm{NA}^{\mathrm{a}}$ & $\begin{array}{c}\text { Flowers, leaves, } \\
\text { juvenile stems }\end{array}$ & Flowering \\
\hline & Protocatechuic acid & $\begin{array}{l}\text { Bahadori et al. } \\
\text { (2018) }\end{array}$ & $\begin{array}{l}\text { 6-7 ug/g dry } \\
\text { extract }^{\text {b }}\end{array}$ & $\mathrm{NA}^{\mathrm{a}}$ & $\begin{array}{c}\text { Flowers, leaves, } \\
\text { juvenile stems }\end{array}$ & Flowering \\
\hline & $\begin{array}{l}\text { Bis-2-ethylhexil- } \\
\text { benzene-1,2- } \\
\text { dicarboxylate }\end{array}$ & Ertaş et al. (2015) & $\begin{array}{l}4 \mathrm{mg} / 1350 \mathrm{~g} \\
\mathrm{dp}\end{array}$ & 2.9 & Aerial parts & Not defined \\
\hline & Anethole & Ertaş et al. (2015) & $\begin{array}{l}0.6 \mathrm{w} / \mathrm{w} \% \text { of } \\
\mathrm{EO}\end{array}$ & 0.3 & Aerial parts & Not defined \\
\hline & Vanillin & $\begin{array}{l}\text { Bahadori et al. } \\
\text { (2018) }\end{array}$ & $\begin{array}{l}\text { 6-31 ug/g dry } \\
\text { extract }^{\mathrm{b}}\end{array}$ & $\mathrm{NA}^{\mathrm{a}}$ & $\begin{array}{l}\text { Flowers, leaves, } \\
\text { juvenile stems }\end{array}$ & Flowering \\
\hline & Eugenol & $\begin{array}{l}\text { Tahira et al. } \\
\text { (2011) }\end{array}$ & $\begin{array}{r}16.79 \mathrm{mg} / \\
100 \mathrm{~g} \mathrm{dp}\end{array}$ & 168 & Leaves & $\begin{array}{l}\text { Spring } \\
\text { vegetative }\end{array}$ \\
\hline
\end{tabular}

${ }^{\mathrm{a} C o n c e n t r a t i o n}$ given without extraction yields, thus in $\mathrm{mg} / \mathrm{kg} \mathrm{dp}$ is not possible to calculate

${ }^{\mathrm{b}}$ Concentration depending on the extraction solvent

${ }^{\mathrm{c}}$ Harvested in June 
Table 4 Flavonoids previously detected in Mentha longifolia and their structure

\begin{tabular}{|c|c|c|c|c|c|c|c|c|c|c|c|}
\hline \multirow[t]{2}{*}{ Subclass } & \multicolumn{9}{|c|}{ Substitution pattern } & \multirow[t]{2}{*}{ Name(s) } & \multirow[t]{2}{*}{$\operatorname{Ref}(*)$} \\
\hline & $\mathrm{C} 3$ & $\mathrm{C} 5$ & C6 & $\mathrm{C} 7$ & $\mathrm{C} 8$ & $\mathrm{C} 2^{\prime}$ & $\mathrm{C} 3^{\prime}$ & $\mathrm{C} 4^{\prime}$ & $\mathrm{C} 5^{\prime}$ & & \\
\hline \multirow[t]{7}{*}{ Flavanones } & $\mathrm{H}$ & $\mathrm{OH}$ & $\mathrm{H}$ & $\mathrm{OH}$ & $\mathrm{H}$ & $\mathrm{H}$ & $\mathrm{H}$ & $\mathrm{OH}$ & $\mathrm{H}$ & Naringenin & 18 \\
\hline & $\mathrm{H}$ & $\mathrm{OH}$ & $\mathrm{H}$ & O-rut & $\mathrm{H}$ & $\mathrm{H}$ & $\mathrm{H}$ & $\mathrm{OH}$ & $\mathrm{H}$ & $\begin{array}{l}\text { Naringenin-7-O-rutinoside, } \\
\text { narirutin }\end{array}$ & 18 \\
\hline & $\mathrm{H}$ & $\mathrm{OH}$ & $\mathrm{H}$ & O-fuc-glu & $\mathrm{H}$ & $\mathrm{H}$ & $\mathrm{OH}$ & $\mathrm{OMe}$ & $\mathrm{H}$ & $\begin{array}{l}\text { 4'-methoxy-naringenin-7-O- } \\
\text { fucopyranosil-1 } \rightarrow 6 \text { - } \\
\text { glucoside, longitin }\end{array}$ & 6 \\
\hline & $\mathrm{H}$ & $\mathrm{OH}$ & $\mathrm{H}$ & $\mathrm{OH}$ & $\mathrm{H}$ & $\mathrm{H}$ & $\mathrm{OH}$ & $\mathrm{OH}$ & $\mathrm{H}$ & Eriodyctiol & 20 \\
\hline & $\mathrm{H}$ & $\mathrm{OH}$ & $\mathrm{H}$ & O-rut & $\mathrm{H}$ & $\mathrm{H}$ & $\mathrm{OH}$ & $\mathrm{OH}$ & $\mathrm{H}$ & $\begin{array}{l}\text { Eriodyctiol-7-O-rutinoside, } \\
\text { eriocitrin }\end{array}$ & $1 ; 18$ \\
\hline & $\mathrm{H}$ & $\mathrm{OH} ?$ & $\mathrm{H}$ & $\mathrm{OH} ?$ & $\mathrm{H}$ & $\mathrm{H}$ & $\mathrm{OH} ?$ & $\mathrm{OH} ?$ & $\mathrm{H}$ & $\begin{array}{l}\text { Eriodyctiol-O-glucoside- } \\
\text { rhamnoside }\end{array}$ & 7 \\
\hline & $\mathrm{H}$ & $\mathrm{OH}$ & $\mathrm{H}$ & O-rut & $\mathrm{H}$ & $\mathrm{H}$ & $\mathrm{OH}$ & $\mathrm{OMe}$ & $\mathrm{H}$ & $\begin{array}{l}\text { Hesperetin-7-O-rutinoside, } \\
\text { hesperidin }\end{array}$ & $1 ; 5$ \\
\hline \multirow[t]{16}{*}{ Flavones } & $\mathrm{H}$ & $\mathrm{OH}$ & $\mathrm{H}$ & $\mathrm{OH}$ & $\mathrm{H}$ & $\mathrm{H}$ & $\mathrm{H}$ & $\mathrm{OH}$ & $\mathrm{H}$ & $\begin{array}{l}\text { 5,7,4'-trihydroxy-flavone, } \\
\text { apigenin }\end{array}$ & $10 ; 15 ; 16 ; 18 ; 20$ \\
\hline & $\mathrm{H}$ & $\mathrm{OH}$ & $\mathrm{H}$ & O-glu & $\mathrm{H}$ & $\mathrm{H}$ & $\mathrm{H}$ & $\mathrm{H}$ & $\mathrm{H}$ & $\begin{array}{l}\text { Apigenin-7-O-glucoside, } \\
\text { cosmosiin, apigetrin }\end{array}$ & 11 \\
\hline & $\mathrm{H}$ & $\mathrm{OH}$ & $\mathrm{H}$ & O-glc & $\mathrm{H}$ & $\mathrm{H}$ & $\mathrm{H}$ & $\mathrm{H}$ & $\mathrm{H}$ & Apigenin-7-O-glucuronide & $1 ; 11$ \\
\hline & $\mathrm{H}$ & $\mathrm{OH}$ & $\mathrm{H}$ & O-rut & $\mathrm{H}$ & $\mathrm{H}$ & $\mathrm{H}$ & $\mathrm{H}$ & $\mathrm{H}$ & $\begin{array}{l}\text { Apigenin-7-O-rutinoside, } \\
\text { isorhoifolin }\end{array}$ & 11 \\
\hline & $\mathrm{H}$ & $\mathrm{OH}$ & $\mathrm{H}$ & $\mathrm{OH}$ & $\mathrm{H}$ & $\mathrm{H}$ & $\mathrm{H}$ & $\begin{array}{l}\mathrm{O}- \\
\text { glu }\end{array}$ & $\mathrm{H}$ & Apigenin-4'-O-glucoside & 14 \\
\hline & $\mathrm{H}$ & O-glu & $\mathrm{H}$ & $\mathrm{OH}$ & $\mathrm{H}$ & $\mathrm{H}$ & $\mathrm{H}$ & $\mathrm{OH}$ & $\mathrm{H}$ & Apigenin-5-O-glucoside & 14 \\
\hline & $\mathrm{H}$ & $\mathrm{OH}$ & C-glu & $\mathrm{OH}$ & $\begin{array}{l}\text { C- } \\
\text { glu }\end{array}$ & $\mathrm{H}$ & $\mathrm{H}$ & $\mathrm{OH}$ & $\mathrm{H}$ & $\begin{array}{l}\text { Apigenin-6,8-C-diglucoside, } \\
\text { vicenin-2 }\end{array}$ & 8 \\
\hline & $\mathrm{H}$ & $\mathrm{OH}$ & $\mathrm{H}$ & $\mathrm{OH}$ & $\mathrm{H}$ & $\mathrm{H}$ & $\mathrm{H}$ & $\mathrm{OMe}$ & $\mathrm{H}$ & $\begin{array}{l}\text { 4'-methoxyapigenin-7-O- } \\
\text { rutinoside, acacetin-7-O- } \\
\text { rutinoside }\end{array}$ & 1 \\
\hline & $\mathrm{H}$ & O-glu & $\mathrm{H}$ & $\mathrm{OMe}$ & $\mathrm{H}$ & $\mathrm{H}$ & $\mathrm{H}$ & $\mathrm{OH}$ & $\mathrm{H}$ & $\begin{array}{l}\text { 7'-methoxyapigenin-5-O- } \\
\text { glucoside, genkwanin-5-O- } \\
\text { glucoside }\end{array}$ & 14 \\
\hline & $\mathrm{H}$ & $\begin{array}{l}\text { O-6"- } \\
\text { mal- } \\
\text { glu }\end{array}$ & $\mathrm{H}$ & $\mathrm{OMe}$ & $\mathrm{H}$ & $\mathrm{H}$ & $\mathrm{H}$ & $\mathrm{OH}$ & $\mathrm{H}$ & $\begin{array}{l}\text { Genkwanin-5-O-[6"-O- } \\
\text { malonyl-]-glucoside }\end{array}$ & 14 \\
\hline & $\mathrm{H}$ & $\mathrm{OH}$ & $\mathrm{H}$ & $\mathrm{OMe}$ & $\mathrm{H}$ & $\mathrm{H}$ & $\mathrm{H}$ & $\mathrm{OH}$ & $\mathrm{H}$ & $\begin{array}{l}\text { Genkwanin-4'-O-glucoside, } \\
\text { fegopolin }\end{array}$ & 14 \\
\hline & $\mathrm{H}$ & $\mathrm{OH}$ & $\mathrm{H}$ & $\mathrm{OH}$ & $\mathrm{H}$ & $\mathrm{H}$ & $\mathrm{OH}$ & $\mathrm{OH}$ & $\mathrm{H}$ & $\begin{array}{l}5,7,3^{\prime}, 4^{\prime} \text {-tetrahydroxy-flavone, } \\
\text { luteolin, luteolol }\end{array}$ & $5 ; 15 ; 16 ; 18 ; 20$ \\
\hline & $\mathrm{H}$ & $\mathrm{OH}$ & $\mathrm{H}$ & O-glu & $\mathrm{H}$ & $\mathrm{H}$ & $\mathrm{OH}$ & $\mathrm{OH}$ & $\mathrm{H}$ & $\begin{array}{l}\text { Luteolin-7-O-glucoside, } \\
\text { cynaroside }\end{array}$ & $1 ; 5 ; 12 ; 16 ; 10$ \\
\hline & $\mathrm{H}$ & $\mathrm{OH}$ & $\mathrm{H}$ & O-glc & $\mathrm{H}$ & $\mathrm{H}$ & $\mathrm{H}$ & $\mathrm{H}$ & $\mathrm{H}$ & Luteolin-7-O-glucuronide & $1 ; 12$ \\
\hline & $\mathrm{H}$ & $\mathrm{OH}$ & $\mathrm{H}$ & O-rut & $\mathrm{H}$ & $\mathrm{H}$ & $\mathrm{H}$ & $\mathrm{H}$ & $\mathrm{H}$ & $\begin{array}{l}\text { Luteolin-7-O-rutinoside, } \\
\text { lonicerin, veronicastroside }\end{array}$ & $1 ; 12$ \\
\hline & $\mathrm{H}$ & $\mathrm{OH}$ & $\mathrm{H}$ & O-neohes & $\mathrm{H}$ & $\mathrm{H}$ & $\mathrm{H}$ & $\mathrm{OH}$ & $\mathrm{H}$ & Luteolin-7-O-neohesperoside & 8 \\
\hline
\end{tabular}


Table 4 continued

\begin{tabular}{|c|c|c|c|c|c|c|c|c|c|c|c|}
\hline \multirow[t]{2}{*}{ Subclass } & \multicolumn{9}{|c|}{ Substitution pattern } & \multirow[t]{2}{*}{ Name(s) } & \multirow{2}{*}{$\begin{array}{l}\text { Ref } \\
(*)\end{array}$} \\
\hline & $\mathrm{C} 3$ & $\mathrm{C} 5$ & C6 & $\mathrm{C} 7$ & $\mathrm{C} 8$ & $\mathrm{C} 2^{\prime}$ & $\mathrm{C} 3^{\prime}$ & $\mathrm{C} 4^{\prime}$ & $\mathrm{C} 5^{\prime}$ & & \\
\hline & $\mathrm{H}$ & $\mathrm{OH}$ & $\mathrm{H}$ & O-neohes & $\mathrm{H}$ & $\mathrm{H}$ & $\mathrm{H}$ & O-soph & $\mathrm{H}$ & $\begin{array}{l}\text { Luteolin-7-O- } \\
\text { neohesperoside-4'-O- } \\
\text { sophoroside }\end{array}$ & 4 \\
\hline & $\mathrm{H}$ & O-glu & $\mathrm{H}$ & $\mathrm{OH}$ & $\mathrm{H}$ & $\mathrm{H}$ & $\mathrm{OH}$ & $\mathrm{OH}$ & $\mathrm{H}$ & $\begin{array}{l}\text { Luteolin-5-O-glucoside, } \\
\text { galuteolin }\end{array}$ & 14 \\
\hline & $\mathrm{H}$ & $\mathrm{OH} ?$ & $\mathrm{H}$ & $\mathrm{OH} ?$ & $\mathrm{H}$ & $\mathrm{H}$ & $\mathrm{OH} ?$ & $\mathrm{OH} ?$ & $\mathrm{H}$ & Luteolin-glucuronide & 7 \\
\hline & $\mathrm{H}$ & $\mathrm{OH} ?$ & $\mathrm{H}$ & $\mathrm{OH} ?$ & $\mathrm{H}$ & $\mathrm{H}$ & $\mathrm{OH} ?$ & $\mathrm{OH} ?$ & $\mathrm{H}$ & $\begin{array}{l}\text { Luteolin-glucuronide, an } \\
\text { other isomer }\end{array}$ & 7 \\
\hline & $\mathrm{H}$ & $\mathrm{OH} ?$ & $\mathrm{H}$ & $\mathrm{OH} ?$ & $\mathrm{H}$ & $\mathrm{H}$ & $\mathrm{OH}$ ? & $\mathrm{OH} ?$ & $\mathrm{H}$ & $\begin{array}{l}\text { Luteolin-glucoside- } \\
\text { rhamnoside }\end{array}$ & 7 \\
\hline & $\mathrm{H}$ & $\mathrm{OH} ?$ & $\mathrm{H}$ & $\mathrm{OH} ?$ & $\mathrm{H}$ & $\mathrm{H}$ & $\mathrm{OH} ?$ & $\mathrm{OH} ?$ & $\mathrm{H}$ & $\begin{array}{l}\text { Methylated luteolin- } \\
\text { glucuronide }\end{array}$ & 7 \\
\hline & $\mathrm{H}$ & $\mathrm{OH} ?$ & $\mathrm{H}$ & $\mathrm{OH} ?$ & $\mathrm{H}$ & $\mathrm{H}$ & $\mathrm{OH} ?$ & $\mathrm{OH} ?$ & $\mathrm{H}$ & Luteolin-diglucuronide & 7 \\
\hline & $\mathrm{H}$ & $\mathrm{OH}$ & $\mathrm{H}$ & O-rut & $\mathrm{H}$ & $\mathrm{H}$ & $\mathrm{H}$ & $\mathrm{OMe}$ & $\mathrm{H}$ & $\begin{array}{l}\text { Diosmetin-7-O-rutinoside, } \\
\text { diosmin }\end{array}$ & 1 \\
\hline & $\mathrm{H}$ & $\mathrm{OH}$ & $\mathrm{H}$ & $\mathrm{OH}$ & $\mathrm{H}$ & $\mathrm{H}$ & $\mathrm{H}$ & $\mathrm{OH}$ & $\mathrm{H}$ & $\begin{array}{l}\text { 5,7,8,4'-tetrahydroxy- } \\
\text { flavone, } 8 \text {-OH-luteolin, } \\
\text { Hypolaetin }\end{array}$ & 8 \\
\hline & $\mathrm{H}$ & $\mathrm{OH}$ & C-glu & $\mathrm{OH}$ & $\begin{array}{l}\text { C- } \\
\text { glu }\end{array}$ & $\mathrm{H}$ & $\mathrm{H}$ & $\mathrm{OH}$ & $\mathrm{H}$ & $\begin{array}{l}\text { Hypolaetin-6,8-C- } \\
\text { diglucoside, lucenin-1 }\end{array}$ & 8 \\
\hline & $\mathrm{H}$ & $\mathrm{OH}$ & $\mathrm{H}$ & $\mathrm{OH}$ & $\mathrm{H}$ & $\mathrm{H}$ & $\mathrm{H}$ & $\mathrm{OMe}$ & $\mathrm{H}$ & Hypolaetin- $4^{\prime}$-methyl ether & 4 \\
\hline & $\mathrm{H}$ & $\mathrm{OH}$ & $\mathrm{H}$ & $\mathrm{OH}$ & $\mathrm{H}$ & $\mathrm{H}$ & O-glu & $\mathrm{OH}$ & $\begin{array}{l}\mathrm{O}- \\
\text { rha }\end{array}$ & $\begin{array}{l}\text { Tricetin- } 3^{\prime} \text {-O-glucoside- } 5^{\prime} \text { - } \\
\text { O-rhamnoside }\end{array}$ & 3 \\
\hline & $\mathrm{H}$ & $\mathrm{OH}$ & $\mathrm{H}$ & $\mathrm{OH}$ & $\mathrm{H}$ & $\mathrm{H}$ & O-rha-rha & $\mathrm{OH}$ & $\mathrm{OH}$ & $\begin{array}{l}\text { Tricetin-3'-O-di- } \\
\text { rhamnoside }\end{array}$ & 3 \\
\hline & $\mathrm{H}$ & $\mathrm{OH}$ & $\mathrm{H}$ & $\mathrm{OMe}$ & $\mathrm{H}$ & $\mathrm{H}$ & O-glu & $\mathrm{OH}$ & $\begin{array}{l}\mathrm{O}- \\
\text { rha }\end{array}$ & $\begin{array}{l}\text { 7-methoxy-tricetin-3'-O- } \\
\text { glucoside-5'-O- } \\
\text { rhamnoside }\end{array}$ & 3 \\
\hline & $\mathrm{H}$ & $\mathrm{OH}$ & $\mathrm{OH}$ & $\mathrm{OMe}$ & $\mathrm{OMe}$ & $\mathrm{H}$ & $\mathrm{OMe}$ & $\mathrm{OH}$ & $\mathrm{H}$ & $\begin{array}{l}5,6,4^{\prime} \text {-trihydroxy-7,8, } 3^{\prime} \text { - } \\
\text { trimethoxy-flavone, } \\
\text { thymonin }\end{array}$ & 5 \\
\hline & $\mathrm{H}$ & $\mathrm{OH}$ & $\mathrm{OMe}$ & $\mathrm{OMe}$ & $\mathrm{OH}$ & $\mathrm{H}$ & $\mathrm{OMe}$ & $\mathrm{OH}$ & $\mathrm{H}$ & $\begin{array}{l}5,8,4^{\prime} \text {-trihydroxy- } 6,7,3^{\prime} \text { - } \\
\text { trimethoxy-flavone }\end{array}$ & 4 \\
\hline & $\mathrm{H}$ & $\mathrm{OH}$ & $\mathrm{OH}$ & $\mathrm{OMe}$ & $\mathrm{OMe}$ & $\mathrm{H}$ & $\mathrm{OMe}$ & $\mathrm{OMe}$ & $\mathrm{H}$ & $\begin{array}{l}\text { 5,6,-dihydroxy-7,8, } 3^{\prime} 4^{\prime}- \\
\text { tetramethoxy-flavone, } \\
\text { pebrellin }\end{array}$ & $2 ; 5$ \\
\hline & $\mathrm{H}$ & $\mathrm{OH}$ & $\mathrm{H}$ & $\mathrm{OMe}$ & $\mathrm{OMe}$ & $\mathrm{OMe}$ & $\mathrm{OMe}$ & $\mathrm{H}$ & $\mathrm{H}$ & $\begin{array}{l}\text { 5-hydroxy-7,8, } 2^{\prime}, 3^{\prime} \text { - } \\
\text { tetramethoxy-flavone }\end{array}$ & 4 \\
\hline & $\mathrm{H}$ & $\mathrm{OH}$ & $\mathrm{OMe}$ & $\mathrm{OH}$ & $\mathrm{H}$ & $\mathrm{OMe}$ & $\mathrm{OMe}$ & $\mathrm{OH}$ & $\mathrm{H}$ & $\begin{array}{l}5,7,4^{\prime} \text {-trihydroxy- } 6,2^{\prime}, 3^{\prime}- \\
\text { trimethoxy-flavone }\end{array}$ & 5 \\
\hline & $\mathrm{H}$ & $\mathrm{OH}$ & $\mathrm{OMe}$ & $\mathrm{OMe}$ & $\mathrm{H}$ & $\mathrm{H}$ & $\mathrm{OMe}$ & $\mathrm{OMe}$ & $\mathrm{H}$ & $\begin{array}{l}\text { 5-hydroxy- } 6,7,3^{\prime}, 4^{\prime}- \\
\text { tetramethoxy-flavone, } \\
\text { belamcanidine }\end{array}$ & 17 \\
\hline & $\mathrm{H}$ & $\mathrm{OH}$ & $\mathrm{OH}$ & $\mathrm{OMe}$ & $\mathrm{H}$ & $\mathrm{H}$ & $\mathrm{OMe}$ & $\mathrm{H}$ & $\mathrm{H}$ & $\begin{array}{l}\text { 5,6,4'-trihydroxy-7,3'- } \\
\text { dimethoxy-flavone }\end{array}$ & 8 \\
\hline \multirow[t]{2}{*}{ Flavonols } & $\mathrm{OH}$ & $\mathrm{OH}$ & $\mathrm{H}$ & $\mathrm{OH}$ & $\mathrm{H}$ & $\mathrm{H}$ & $\mathrm{H}$ & $\mathrm{OH}$ & $\mathrm{H}$ & Kaempferol & $19 ; 21$ \\
\hline & O-glu & $\mathrm{OH}$ & $\mathrm{H}$ & $\mathrm{OH}$ & $\mathrm{H}$ & $\mathrm{H}$ & $\mathrm{H}$ & $\mathrm{OH}$ & $\mathrm{H}$ & $\begin{array}{l}\text { Kaempferol-3-O-glucoside, } \\
\text { astragalin }\end{array}$ & $10 ; 14$ \\
\hline
\end{tabular}


Table 4 continued

\begin{tabular}{|c|c|c|c|c|c|c|c|c|c|c|c|}
\hline \multirow[t]{2}{*}{ Subclass } & \multicolumn{9}{|c|}{ Substitution pattern } & \multirow[t]{2}{*}{ Name(s) } & \multirow[t]{2}{*}{$\operatorname{Ref}(*)$} \\
\hline & $\mathrm{C} 3$ & $\mathrm{C} 5$ & C6 & $\mathrm{C} 7$ & $\mathrm{C} 8$ & $\mathrm{C} 2^{\prime}$ & $\mathrm{C} 3^{\prime}$ & $\mathrm{C} 4^{\prime}$ & $\mathrm{C} 5^{\prime}$ & & \\
\hline & O-soph & $\mathrm{OH}$ & $\mathrm{H}$ & $\mathrm{OH}$ & $\mathrm{H}$ & $\mathrm{H}$ & $\mathrm{H}$ & $\mathrm{OH}$ & $\mathrm{H}$ & $\begin{array}{l}\text { Kaempferol-3-O-sophoroside, } \\
\text { sophoraflavonoside }\end{array}$ & 14 \\
\hline & O-rha & $\mathrm{OH}$ & $\mathrm{H}$ & $\mathrm{OH}$ & $\mathrm{H}$ & $\mathrm{H}$ & $\mathrm{H}$ & $\mathrm{OH}$ & $\mathrm{H}$ & Kaempferol-3-O-rhamnoside, afzelin & 14 \\
\hline & $\mathrm{OH}$ & $\mathrm{OH}$ & $\mathrm{H}$ & O-rha & $\mathrm{H}$ & $\mathrm{H}$ & $\mathrm{H}$ & $\mathrm{OH}$ & $\mathrm{H}$ & Kaempferol-7-O-rhamnoside & 14 \\
\hline & $\begin{array}{c}\text { O-6"- } \\
\text { mal- } \\
\text { glu }\end{array}$ & $\mathrm{OH}$ & $\mathrm{H}$ & O-rha & $\mathrm{H}$ & $\mathrm{H}$ & $\mathrm{H}$ & $\mathrm{OH}$ & $\mathrm{H}$ & $\begin{array}{l}\text { Kaempferol-3-O-[6"-O-malonyl-]- } \\
\text { glucoside-7-O-rhamnoside }\end{array}$ & 14 \\
\hline & $\mathrm{OH}$ & $\mathrm{OH}$ & $\mathrm{H}$ & $\mathrm{OH}$ & $\mathrm{H}$ & $\mathrm{H}$ & $\mathrm{OH}$ & $\mathrm{OH}$ & $\mathrm{H}$ & Quercetin & $16 ; 19 ; 21$ \\
\hline & O-glu & $\mathrm{OH}$ & $\mathrm{H}$ & $\mathrm{OH}$ & $\mathrm{H}$ & $\mathrm{H}$ & $\mathrm{OH}$ & $\mathrm{OH}$ & $\mathrm{H}$ & Quercetin-3-O-glucoside, isoquercitrin & $10 ; 15$ \\
\hline & O-rut & $\mathrm{OH}$ & $\mathrm{H}$ & $\mathrm{OH}$ & $\mathrm{H}$ & $\mathrm{H}$ & $\mathrm{OH}$ & $\mathrm{OH}$ & $\mathrm{H}$ & Quercetin-3-O-rutinoside, rutin & $15 ; 18 ; 19 ; 20 ; 21$ \\
\hline & O-glu & $\mathrm{OH}$ & $\mathrm{H}$ & O-glu & $\mathrm{H}$ & $\mathrm{H}$ & $\mathrm{OH}$ & $\mathrm{OH}$ & $\mathrm{H}$ & Quercetin-3,7-O-diglucoside & $10 ; 15$ \\
\hline & $\mathrm{OH}$ & $\mathrm{OH}$ & $\mathrm{H}$ & O-rha & $\mathrm{H}$ & $\mathrm{H}$ & $\mathrm{OH}$ & $\mathrm{OMe}$ & $\mathrm{OH}$ & 4'-methoxymyricetin-3-O-rhamnoside & 4 \\
\hline
\end{tabular}

fuc Fucose, glu Glucose, glc Glucuronic acid, neohes Neohesperidose, rut Rutinose, rha Rhamnose, soph Sophorose, mal Malonyl, OMe Methoxyl.'?' No information is provided about the site of the glycosidic bond(s)

*For the sake of transparency, References in this table are numbered: (1) Bourwieg and Pohl (1973); (2) Tomás-Barberán et al. (1988); (3) Sharaf et al. (1999); (4) Jahan et al. (2001), (5) Ghoulami et al. (2001); (6) Ali et al. (2002); (7) Krzyzanowska et al. (2011); (8) Ulubelen et al. (2005); (9) Fialová et al. (2008); (10) Akroum et al. (2009); (11) Baris et al. (2011); (12) Orhan et al. (2012); (13) Pereira and Cardoso (2013); (14) Stanislavljević et al. (2012); (15) Benedec et al. (2013); (16) Elansary and Mahmoud (2015); (17) Ertaş et al. (2015); (18) Hawrył et al. (2016); (19) Patonay et al. (2017); (20) Bahadori et al. (2018) (21) Park et al. (2019)

from a Pakistani sample may be mentioned as a novelty (Ali et al. 2002).

\section{Flavones}

Table 5 shows the available quantitative data of flavones in M. longifolia. This flavonoid subclass shows a very wide variability in ML samples. Among them, there are some compounds which have not been known before and detected especially in ML for the first time. A novel aglycone with unconventional substitution pattern, 5,7,4'-trihydroxy-6,2' $3^{\prime}$-trimethoxy-flavone, was detected by Ghoulami et al. (2001) from Morocco. Besides, a low concentration of another new aglycone, 5,8, $4^{\prime}$-trihydroxy-6,7,3'-trimethoxy-flavone was found by Jahan et al. (2001), from Pakistan. Exploration of three previously unknown tricetin derivatives in a M. longifolia sample from Saudi Arabia is reported by Sharaf et al. (1999). According to the authors, it is the first report on flavones bearing trisubstituted B ring in the whole Lamiaceae family. However, the occurrence of the mentioned special flavones in horsemint seems to be supported only by the cited single reference.
Among flavone glycosides, cynaroside has been detected repeatedly, (Table 4) although its concentration is low (or not provided) (Table 5). In some cases, the sites of the glycosidic bonds are not designated, thus the exact glycoside molecule remains questionable, e.g. luteolin-glucorhamnoside and luteolin-glucuronides in study of Krzyzanowska et al. (2011). It can be established, that the sporadic data about flavone-7-O-glycosides as summarized in Tables 4 and 5 do not seem to represent strong support for the universal and frequent accumulation of them in horsemint, although these ingredients have been frequently described in other mint species (Guédon and Pasquier 1994; Areias et al. 2001; DamienDorman et al. 2003a, b; Koşar et al. 2004).

\section{Flavonols}

Although the previous reviews (Pereira and Cardoso 2013; Mikaili et al. 2013; Farzaei et al. 2017) do not deal with this subclass in detail when discussing the flavonoids of $M$. longifolia, the available literature shows that flavonols may frequently be present in this species. Quercetin and kaempferol together with their 
Table 5 Available quantitative data of flavones in Mentha longifolia L samples

\begin{tabular}{|c|c|c|c|c|c|}
\hline \multirow[t]{2}{*}{ Name } & \multirow[t]{2}{*}{ References } & \multicolumn{2}{|l|}{ Quantitative data } & \multirow{2}{*}{$\begin{array}{l}\text { Plant part } \\
\text { investigated }\end{array}$} & \multirow[t]{2}{*}{ Phenophase } \\
\hline & & $\begin{array}{l}\text { Concentration } \\
\text { measured }\end{array}$ & $\begin{array}{l}\text { Concentration } \\
(\mathrm{mg} / \mathrm{kg} \mathrm{dp})\end{array}$ & & \\
\hline $\begin{array}{l}5,7,4^{\prime} \text {-trihidroxi-flavone, } \\
\text { apigenin }\end{array}$ & $\begin{array}{l}\text { Elansary and } \\
\text { Mahmoud (2015) }\end{array}$ & $\begin{array}{l}3.86 \mathrm{mg} / \mathrm{g} \text { dry } \\
\text { extract }\end{array}$ & $\mathrm{NA}^{\mathrm{a}}$ & Leaves & Not defined \\
\hline $\begin{array}{l}5,7,4^{\prime} \text {-trihidroxi-flavone, } \\
\text { apigenin }\end{array}$ & $\begin{array}{l}\text { Patonay et al. } \\
\text { (2017) }\end{array}$ & $\begin{array}{l}19.7-144.2 \mathrm{mg} / \mathrm{kg} \\
\mathrm{dp}\end{array}$ & $19.7-144.2^{\mathrm{c}}$ & $\begin{array}{l}\text { Leaves, stem, } \\
\text { inflorescence }\end{array}$ & Flowering \\
\hline $\begin{array}{l}5,7,4^{\prime} \text {-trihidroxi-flavone, } \\
\text { apigenin }\end{array}$ & $\begin{array}{l}\text { Bahadori et al. } \\
\text { (2018) }\end{array}$ & $\begin{array}{l}\text { 94-124 ug/g dry } \\
\text { extract }^{\mathrm{c}}\end{array}$ & $\mathrm{NA}^{\mathrm{a}}$ & $\begin{array}{l}\text { Flowers, leaves, } \\
\text { juvenile stems }\end{array}$ & Flowering \\
\hline $\begin{array}{l}\text { Apigenin-7-O-glucoside, } \\
\text { cosmosiin, apigetrin }\end{array}$ & Baris et al. (2011) & $\begin{array}{l}3.6 \mathrm{mg} \text { isolated } \\
\text { from } 1 \mathrm{~kg} \text { sample }\end{array}$ & 3.6 & Leaves, stem & Flowering \\
\hline Apigenin-7-O-glucuronide & Baris et al. (2011) & $\begin{array}{l}5.2 \mathrm{mg} \text { isolated } \\
\text { from } 1 \mathrm{~kg} \text { sample }\end{array}$ & 5.2 & Leaves, stem & Flowering \\
\hline $\begin{array}{l}\text { Apigenin-7-O-rutinoside, } \\
\text { isorhoifolin }\end{array}$ & Baris et al. (2011) & $\begin{array}{l}6.3 \mathrm{mg} \text { isolated } \\
\text { from } 1 \mathrm{~kg} \text { sample }\end{array}$ & 6.3 & Leaves, stem & Flowering \\
\hline Apigenin- $4^{\prime}-\mathrm{O}$-glucoside & $\begin{array}{l}\text { Stanislavljević et al. } \\
\text { (2012) }\end{array}$ & $0.81 \mathrm{mg} / \mathrm{g}$ extract & 191.0 & $\begin{array}{l}\text { Above-ground } \\
\text { parts }\end{array}$ & Flowering \\
\hline Apigenin-5-O-glucoside & $\begin{array}{l}\text { Stanislavljević et al. } \\
\text { (2012) }\end{array}$ & $7.53 \mathrm{mg} / \mathrm{g}$ extract & 843.0 & $\begin{array}{l}\text { Above-ground } \\
\text { parts }\end{array}$ & Flowering \\
\hline $\begin{array}{l}5,7,3^{\prime}, 4^{\prime} \text {-tetrahidroxi-flavone, } \\
\text { luteolin }\end{array}$ & $\begin{array}{l}\text { Benedec et al. } \\
\text { (2013) }\end{array}$ & $1.764 \mathrm{mg} / \mathrm{g} \mathrm{dp}$ & 17.6 & Aerial parts & Flowering \\
\hline $\begin{array}{l}5,7,3^{\prime}, 4^{\prime} \text {-tetrahidroxi-flavone, } \\
\text { luteolin }\end{array}$ & $\begin{array}{l}\text { Elansary and } \\
\text { Mahmoud (2015) }\end{array}$ & $\begin{array}{l}3.21 \mathrm{mg} / \mathrm{g} \text { dry } \\
\text { extract }\end{array}$ & $\mathrm{NA}^{\mathrm{a}}$ & Leaves & Not defined \\
\hline $\begin{array}{l}5,7,3^{\prime}, 4^{\prime} \text {-tetrahidroxi-flavone, } \\
\text { luteolin }\end{array}$ & $\begin{array}{l}\text { Bahadori et al. } \\
\text { (2018) }\end{array}$ & $\begin{array}{l}84-162 \mathrm{ug} / \mathrm{g} \text { dry } \\
\text { ectracr }^{\mathrm{c}}\end{array}$ & $\mathrm{NA}^{\mathrm{a}}$ & $\begin{array}{l}\text { Flowers, leaves, } \\
\text { juvenile stems }\end{array}$ & Flowering \\
\hline $\begin{array}{l}\text { Luteolin-7-O-glucoside, } \\
\text { cynaroside }\end{array}$ & $\begin{array}{l}\text { Elansary and } \\
\text { Mahmoud (2015) }\end{array}$ & $\begin{array}{l}3.91 \mathrm{mg} / \mathrm{g} \text { dry } \\
\text { extract }\end{array}$ & $\mathrm{NA}^{\mathrm{a}}$ & Leaves & Not defined \\
\hline $\begin{array}{l}\text { Luteolin-7-O-glucoside, } \\
\text { cynaroside }\end{array}$ & Orhan et al. (2012) & $\begin{array}{l}7.0 \mathrm{mg} \text { isolated } \\
\text { from } 1 \mathrm{~kg} \text { sample }\end{array}$ & 7.0 & $\begin{array}{l}\text { Leaves, stem, } \\
\text { inflorescence }\end{array}$ & Flowering \\
\hline Luteolin-7-O-glucuronide & Orhan et al. (2012) & $\begin{array}{l}4.0 \mathrm{mg} \text { isolated } \\
\text { from } 1 \mathrm{~kg} \text { sample }\end{array}$ & 4.0 & $\begin{array}{l}\text { Leaves, stem, } \\
\text { inflorescence }\end{array}$ & Flowering \\
\hline $\begin{array}{l}\text { Luteolin-7-O-rutinoside, } \\
\text { lonicerin, veronicastroside }\end{array}$ & Orhan et al. (2012) & $\begin{array}{l}18.3 \mathrm{mg} \text { isolated } \\
\text { from } 1 \mathrm{~kg} \text { sample }\end{array}$ & 18.3 & $\begin{array}{l}\text { Leaves, stem, } \\
\text { inflorescence }\end{array}$ & Flowering \\
\hline $\begin{array}{l}\text { Luteolin-5-O-glucoside, } \\
\text { galuteolin }\end{array}$ & $\begin{array}{l}\text { Stanislavljević et al. } \\
\text { (2012) }\end{array}$ & $1.69 \mathrm{mg} / \mathrm{g}$ extract. ${ }^{\mathrm{d}}$ & 196 & $\begin{array}{l}\text { Above-ground } \\
\text { parts) }\end{array}$ & Flowering \\
\hline Luteolin-glucuronide & $\begin{array}{l}\text { Krzyzanowska et al. } \\
\text { (2011) }\end{array}$ & $2.237 \mathrm{mg} / \mathrm{g} \mathrm{dp}$ & 2237 & $\begin{array}{l}\text { Not defined (field } \\
\text { plant) }\end{array}$ & Not defined \\
\hline Luteolin-glucuronide & $\begin{array}{l}\text { Krzyzanowska et al. } \\
\text { (2011) }\end{array}$ & $0.007 \mathrm{mg} / \mathrm{g} \mathrm{dp}$ & 7.0 & $\begin{array}{l}\text { Not defined } \\
\quad \text { (in vitro plant) }\end{array}$ & Not defined \\
\hline Luteolin-glucuronide & $\begin{array}{l}\text { Krzyzanowska et al. } \\
\text { (2011) }\end{array}$ & traces $\mathrm{mg} / \mathrm{g} \mathrm{dp}$ & - & Cell suspension & $(-)$ \\
\hline $\begin{array}{l}\text { Luteolin-glucuronide, an other } \\
\text { isomer }\end{array}$ & $\begin{array}{l}\text { Krzyzanowska et al. } \\
\text { (2011) }\end{array}$ & $0.285 \mathrm{mg} / \mathrm{g} \mathrm{dp}$ & 285 & $\begin{array}{l}\text { Not defined (field } \\
\text { plant) }\end{array}$ & Not defined \\
\hline $\begin{array}{l}\text { Luteolin-glucuronide, an other } \\
\text { isomer }\end{array}$ & $\begin{array}{l}\text { Krzyzanowska et al. } \\
\text { (2011) }\end{array}$ & $0.005 \mathrm{mg} / \mathrm{g} \mathrm{dp}$ & 5.0 & $\begin{array}{l}\text { Not defined } \\
\quad \text { (in vitro plant) }\end{array}$ & Not defined \\
\hline $\begin{array}{l}\text { Luteolin-glucuronide, an other } \\
\text { isomer }\end{array}$ & $\begin{array}{l}\text { Krzyzanowska et al. } \\
\text { (2011) }\end{array}$ & $0.074 \mathrm{mg} / \mathrm{g} \mathrm{dp}$ & 74.0 & Callus culture & $(-)$ \\
\hline Luteolin-glucoside-rhamnoside & $\begin{array}{l}\text { Krzyzanowska et al. } \\
\text { (2011) }\end{array}$ & $3.576 \mathrm{mg} / \mathrm{g} \mathrm{dp}$ & 3576 & $\begin{array}{l}\text { Not defined (field } \\
\text { plant) }\end{array}$ & Not defined \\
\hline
\end{tabular}


Table 5 continued

\begin{tabular}{|c|c|c|c|c|c|}
\hline \multirow[t]{2}{*}{ Name } & \multirow[t]{2}{*}{ References } & \multicolumn{2}{|l|}{ Quantitative data } & \multirow{2}{*}{$\begin{array}{l}\text { Plant part } \\
\text { investigated }\end{array}$} & \multirow[t]{2}{*}{ Phenophase } \\
\hline & & $\begin{array}{l}\text { Concentration } \\
\text { measured }\end{array}$ & $\begin{array}{l}\text { Concentration } \\
(\mathrm{mg} / \mathrm{kg} \mathrm{dp})\end{array}$ & & \\
\hline Luteolin-glucoside-rhamnoside & $\begin{array}{l}\text { Krzyzanowska et al. } \\
\text { (2011) }\end{array}$ & $1.134 \mathrm{mg} / \mathrm{g} \mathrm{dp}$ & 1134 & $\begin{array}{l}\text { Not defined } \\
\text { (in vitro plant) }\end{array}$ & Not defined \\
\hline Luteolin-glucoside-rhamnoside & $\begin{array}{l}\text { Krzyzanowska et al. } \\
\text { (2011) }\end{array}$ & $0.018 \mathrm{mg} / \mathrm{g} \mathrm{dp}$ & 13.0 & Cell suspension & $(-)$ \\
\hline Luteolin-glucoside-rhamnoside & $\begin{array}{l}\text { Krzyzanowska et al. } \\
\text { (2011) }\end{array}$ & $0.013 \mathrm{mg} / \mathrm{g} \mathrm{dp}$ & 18.0 & Callus culture & $(-)$ \\
\hline Methylated luteolin glucuronide & $\begin{array}{l}\text { Krzyzanowska et al. } \\
\text { (2011) }\end{array}$ & $0.007 \mathrm{mg} / \mathrm{g} \mathrm{dp}$ & 7.0 & $\begin{array}{l}\text { Not defined (field } \\
\text { plant) }\end{array}$ & Not defined \\
\hline Methylated luteolin glucuronide & $\begin{array}{l}\text { Krzyzanowska et al. } \\
\text { (2011) }\end{array}$ & $0.013 \mathrm{mg} / \mathrm{g} \mathrm{dp}$ & 13.0 & $\begin{array}{l}\text { Not defined } \\
\text { (in vitro plant) }\end{array}$ & Not defined \\
\hline Luteolin-diglucuronide & $\begin{array}{l}\text { Krzyzanowska et al. } \\
\text { (2011) }\end{array}$ & $1.432 \mathrm{mg} / \mathrm{g} \mathrm{dp}$ & 1432 & $\begin{array}{l}\text { Not defined (field } \\
\text { plant) }\end{array}$ & Not defined. \\
\hline $\begin{array}{l}\text { Tricetin- } 3^{\prime} \text {-O-glucoside }-5^{\prime}-\mathrm{O}- \\
\text { rhamnoside }\end{array}$ & Sharaf et al. (1999) & $\begin{array}{l}28 \mathrm{mg} \text { isolated from } \\
200 \mathrm{~g} \mathrm{dp}\end{array}$ & 140 & Aerial parts & Not defined \\
\hline Tricetin-3'-O-di-rhamnoside & Sharaf et al. (1999) & $\begin{array}{l}31 \mathrm{mg} \text { isolated from } \\
200 \mathrm{~g} \mathrm{dp}\end{array}$ & 155 & Aerial parts & Not defined \\
\hline $\begin{array}{l}\text { 7-methoxy-tricetin-3'-O- } \\
\text { glucoside-5'-O-rhamnoside }\end{array}$ & Sharaf et al. (1999) & $\begin{array}{l}21 \mathrm{mg} \text { isolated from } \\
200 \mathrm{~g} \mathrm{dp}\end{array}$ & 105 & Aerial parts & Not defined \\
\hline Genkwanin-5-O-glucoside & $\begin{array}{l}\text { Stanislavljevićet al. } \\
\text { (2012) }\end{array}$ & $0.56 \mathrm{mg} / \mathrm{g}$ extract & 64.9 & $\begin{array}{l}\text { Above-ground } \\
\text { parts }\end{array}$ & Flowering \\
\hline $\begin{array}{l}\text { Genkwanin-5-O-[6"-O- } \\
\text { malonyl-]-glucoside }\end{array}$ & $\begin{array}{l}\text { Stanislavljević et al. } \\
\text { (2012) }\end{array}$ & $1.96 \mathrm{mg} / \mathrm{g}$ extract & 183. & $\begin{array}{l}\text { Above-ground } \\
\text { parts }\end{array}$ & Flowering \\
\hline $\begin{array}{l}\text { Genkwanin-4'-O-glucoside, } \\
\text { fegopolin }\end{array}$ & $\begin{array}{l}\text { Stanislavljević et al. } \\
\text { (2012) }\end{array}$ & $\begin{array}{l}0.52-0.57 \mathrm{mg} / \mathrm{g} \\
\operatorname{extract}^{\mathrm{d}}\end{array}$ & $58.8-60.3$ & $\begin{array}{l}\text { Above-ground } \\
\text { parts }\end{array}$ & Flowering \\
\hline $\begin{array}{l}\text { 5,6,-dihydroxy- } 7,8,3^{\prime} 4^{\prime}- \\
\text { tetrametoxi-flavone, pebrellin }\end{array}$ & $\begin{array}{l}\text { Ghoulami et al. } \\
\text { (2001) }\end{array}$ & 0.015 w/w $\% \mathrm{dp}$ & 150 & Aerial parts & $\begin{array}{l}\text { End of } \\
\text { vegetative } \\
\text { cycle }\end{array}$ \\
\hline $\begin{array}{l}5,7,4^{\prime} \text {-trihydroxy- } 6,2^{\prime}, 3^{\prime}- \\
\text { trimetoxi-flavone }\end{array}$ & $\begin{array}{l}\text { Ghoulami et al. } \\
\text { (2001) }\end{array}$ & $0.010 \mathrm{w} / \mathrm{w} \% \mathrm{dp}$ & 100 & Aerial parts & $\begin{array}{l}\text { End of } \\
\text { vegetative } \\
\text { cycle }\end{array}$ \\
\hline $\begin{array}{l}\text { 5-hydroxy- } 6,7,3^{\prime}, 4^{\prime}- \\
\text { tetrametoxi-flavone, } \\
\text { belamcanidine }\end{array}$ & Ertaş et al. (2015) & $\begin{array}{l}5 \mathrm{mg} \text { isolated from } \\
1350 \mathrm{~g} \mathrm{dp}\end{array}$ & 3.7 & Not defined & Not defined \\
\hline $\begin{array}{l}\text { 5-hydroxy-7,8, } 2^{\prime}, 3^{\prime}- \\
\text { tetramethoxy-flavone }\end{array}$ & Jahan et al. (2001) & $\begin{array}{l}25 \mathrm{mg} \text { isolated from } \\
20 \mathrm{~kg} \mathrm{dp}\end{array}$ & $1.25 \times 10^{-3}$ & Not defined & Not defined ${ }^{\mathrm{d}}$ \\
\hline Hypolaetin- $4^{\prime}$ methyl ether & Jahan et al. (2001) & $\begin{array}{l}30 \mathrm{mg} \text { isolated from } \\
20 \mathrm{~kg} \mathrm{dp}\end{array}$ & $1.5 \times 10^{-3}$ & Not defined & Not defined ${ }^{\mathrm{d}}$ \\
\hline $\begin{array}{l}5,8,4^{\prime} \text {-trihydroxy- } 6,7,3^{\prime}- \\
\text { trimethoxy-flavone }\end{array}$ & Jahan et al. (2001) & $\begin{array}{l}45 \mathrm{mg} \text { from } 20 \mathrm{~kg} \\
\mathrm{dp}\end{array}$ & $2.25 \times 10^{-3}$ & Not defined & Not defined ${ }^{\mathrm{d}}$ \\
\hline
\end{tabular}

${ }^{\mathrm{a}}$ Concentration given in $\mathrm{mg} / \mathrm{g}$ extracts without providing yields, thus in $\mathrm{mg} / \mathrm{kg} \mathrm{dp}$. is not possible to calculate

${ }^{\mathrm{b}}$ Concentrations depending on the extraction solvent

${ }^{\mathrm{c}}$ Concentrations depending on drying method

${ }^{\mathrm{d}}$ Harvested in March 
glycosides are most often reported from $M$. longifolia (Table 4.). The concentration ranges are variable, like in the case of rutin: $0.822 \mathrm{mg} / 100 \mathrm{~g} \mathrm{dp}$ (Benedec et al. 2013) or $1166 \mathrm{mg} / 100 \mathrm{~g}$ dp (Park et al. 2019). Flavonol-rich samples were reported from Serbia (Stanislavljević et al. 2012), Hungary (Patonay et al. 2017) and Korea (Park et al. 2019). Interestingly, Stanislavljević et al. (2012) reported astragalin to be the dominant flavonol constituent of a $M$. longifolia charge $(61.36 \mathrm{mg} / \mathrm{g}$ extract calculated with yield: $7118 \mathrm{mg} / \mathrm{kg} \mathrm{dp}$ ).

It seems, that the actual amount of flavonoid compounds in the drug may be influenced by drying method (Stanislavljević et al. 2012) or other postharvest treatments like heating the fresh plant material (Stocker and Pohl 1976). These questions may need a further study.

\section{Antiradical and antimicrobial properties of phenolics occurring in horsemint}

Rosmarinic acid plays an important role in the antioxidant properties of $M$. longifolia extracts. Dudai and co-workers established a tight correlation $\left(R^{2}=0.38\right)$ between rosmarinic acid content and results of DPPH assay. However, Fialová and coworkers (2008) suggest, that other constituent(s) than this may play a role in the radical scavenging activity of $M$. longifolia as the maxima of THD, TF and antiradical activity do not coincide with the maxima of RA content. Interestingly, the concentration of caffeic acid does not seem to correlate with results of DPPH assay $\left(R^{2}=0.0119\right)$ contrary to its known AO efficiency (Koşar et al. 2004; Csepregi et al. 2016). Grayer et al. (2003) observed nepetoidin B to be a stronger AO than gallic acid in DPPH assay.

As Table 4 shows, a significant proportion of the flavonoids detected in ML are the 7-O-glycosides. Although they are frequent in antioxidant-rich species of plant families e.g. Lamiaceae, Apiaceae, Asteraceae, their $\mathrm{AO}$ properties are less known in comparison with 3-O-glycosides (Csepregi et al. 2016). Therefore, the antiradical abilities of 7-O-glycosides may principally be outlined using studies of structureactivity relationship. Bors and co-workers (1990) studied the kinetics of various flavonoids against $\mathrm{OH}, \mathrm{N}_{3}$ and tert-butoxyl radicals demonstrating that the key of the AO activity of flavonoids towards radicals is the ability to form a longlife secondary aroxyl radical which could take part in recombinations. In this consideration, authors outlined the necessary structural traits providing better delocalization of the unpaired electron and in consequence, stability of aroxyl radicals. These are the followings (1) free ortho-dihydroxy group at B ring (catechol moiety) (2) the free -OH group at $\mathrm{C} 3$ (3) double bond at $\mathrm{C} 2-\mathrm{C} 3$ and carbonyl on $\mathrm{C} 4$, because of conjugation (4) additionally, presence of free $-\mathrm{OH}$ groups at $\mathrm{C} 5$ and C7. Later, studies ranking flavonoids on TEAC (Rice-Evans et al. 1996; Csepregi et al. 2016) and DPPH assays (Burda and Ołeszek 2001; Csepregi et al. 2016) modified this idea. Catechol moiety was repeatedly observed to play a key role in $\mathrm{AO}$ properties, followed by free $\mathrm{C} 3-\mathrm{OH}$. The latter was recently observed to be tightly and significantly correlated with activity in TEAC and FRAP assays but loosely coupled to the activity in DPPH and FolinCiocalteu's assay (Csepregi et al. 2016). The C2-C3$\mathrm{C} 4$ system was reinforced to function only in combination with free catechol moiety and/or C3-OH (Wen et al. 2014; Csepregi et al. 2016). Based on these considerations, some flavonoid-7-O-glycosides detected in ML may deserve attention. Thus, luteolin-7-O-glycosides may be predicted as active against some radicals as rutin as they have catechol moiety and $\mathrm{C} 2-\mathrm{C} 3-\mathrm{C} 4$ conjugation but free $\mathrm{C} 3-\mathrm{OH}$ is absent. A ranking of flavonoids by activity against DPPH (Burda and Ołeszek 2001) supports this idea. Here, rutin showed 90.9 IC\% and cynaroside 87.6 IC\%. Luteolin itself was also observed to show antiradical activity stronger than of BHT on DPPH assay but weaker efficacy on ORAC (Wen et al. 2014), suggesting that the lack of $\mathrm{C} 3-\mathrm{OH}$ might decrease this kind of AO activity. On the other hand, eriodyctiol and 7-O-glycosides may be considered as stronger antiradical agents than other flavanones of ML because only they have a free catechol group. Damien-Dorman and co-workers (2003a, b) declared, that mints richest in eriocitrin and rich in RA showed the highest activity against DPPH and $\mathrm{OH}$. Their further study (Koşar et al. 2004) demonstrated a high correlation between DPPH antiradical activity of Mentha extracts and concentration of caffeic acid, rosmarinic acid, lonicerin, eriocitrin and an undefined luteolin-7-O-glycoside. Antiradical activity of luteolin-5-O-glycosides like galuteolin may be supposed to be similar to 7-Oanalogues because of the presence of free catechol 
moiety and $\mathrm{C} 2-\mathrm{C} 3-\mathrm{C} 4$ conjugation. Naringenin and apigenin derivatives however, as it may be expected based on their structure, did not show this response.

It must be emphasized, that synergistic effects between some flavonoids and/or flavonoids and caffeic acid derivatives may occur, depending on the ratio of concentration and their redox potential and the presence of catechol moiety in the case of flavonoids (Freeman et al. 2010; Reber et al. 2011; OłszowyTomczyk 2020). A very recent long-needed review of Ołszowy-Tomczyk (2020) called attention to the mutual effects of plant phenolics in binary mixtures. The detailed data collected by the author shows that there are some cases when synergistic or additive effects were reported between polyphenols e.g. between rosmarinic acid and quercetin in the case of AAPH induced oxidation; between chlorogenic acid and hesperidin, also between p-coumaric acid and quercetin in ORAC assay. On the other side, no antagonistic effect was reported to rosmarinic acid and flavonoids except an observation on FRAP assay of rutin and rosmarinic acid (Hajimehdipoor et al. 2014). Although an extract is much more complex than a binary mixture, synergistic or antagonistic effects may be considered when the background of antioxidant properties of a ML extract is studied.

Beside the plant material itself, studies rarely focused on other factors which might influence the AO properties of ML extracts. Fialová and co-workers (2008) proved that ML show higher AO activity, THD and TF in July than in September (DPPH EC 50 in July $24.60 \mu \mathrm{g} / \mathrm{mL}$, in September $45.20 \mu \mathrm{g} / \mathrm{mL}$ ). Further studies are needed in this respect.

Focusing on the food preservative utilisation of $M$. longifolia, beyond the AO activity of phenolic compounds, the activity against bacteria or fungi causing food spoilage and/or foodborne diseases may be taken into account. Akroum and co-workers (2009) established that isoquercitrin in $M$. longifolia showed the strongest growth inhibitory effect against B.cereus, B.subtilis, S.aureus, E.coli and P.aeruginosa (MIC = $0.03-0.09 \mu \mathrm{g} / \mathrm{mL}$ ). Synergism among these molecules was observed. Other polyphenols of ML may also be potential antimicrobial agents, as documented in in vitro studies in the case of other species, like apigenin (Basile et al. 1999; Metsämuuren and Sirén 2019), luteolin (Wen et al. 2014) and nepetoidins (Grayer et al. 2003).

\section{The volatile composition of horsemint}

Essential oil (EO) content of horsemint and classification of its constituents

According to recent data, volatile components accumulate in $M$. longifolia in a range of $0.5-1 \%$ dry weight (Hajlaoui et al. 2009; Sharopov et al. 2012; Iqbal et al. 2013; Llorens-Molina et al. 2015; Kapp 2015). However, earlier studies report significantly higher EO contents, up to 1.6-2.8\% from Eastern Crete (Karousou et al. 1998) and 3.8\% from Sinai (Fleisher and Fleisher 1991). This wide interval of EO contents may be in part coupled to sampling methods and the varying phenological phase or organic composition of the plants (EO yield of the plant is recently observed by Llorens-Molina et al. (2020) to reach its maximum in advanced flowering stage). Anyhow, the different experimental conditions make a proper evaluation difficult. Illustrating this, the analysed sample types include flowering shoots (Karousou et al. 1998), shoots at the end of flowering, or seed ripening stage (Baser et al. 1999) or even leaves separated from the stems (Orav et al. 2013).

Volatiles of ML show extraordinary wide variability, involving multiple metabolic pathways. Based on works of Başer and co-authors (1999; 2012), volatile terpenes of $M$. longifolia could be perspicuously grouped by structure (Figs. 1,2). These groups and their important representants are presented below.

\section{Open-chain monoterpenes}

Linalool (Mimica-Dukic and Bozin 2008) and linalyl acetate may be present in concentrations above $10 \%$ of ML EO (Al-Okbi et al. 2015), although they do not appear in all M. longifolia samples. Thus, they may not be considered as universal constituent of the species. Myrcene was also reported in concentration around $10 \%$ in samples from Lithuania (Venskutonis 1996).

\section{Limonene and its 2-oxo derivatives}

Carvone, dihydrocarvone, cis- and trans-carvyl acetate, cis- and trans-dihydrocarveol frequently appear in EOs of M. longifolia (Başer et al. 1999; Sharopov et al. 2012; Mimica-Dukic and Bozin 2008). 55-66\% carvone was present in the EO of the samples from Crete (Karousou et al. 1998) while 50-65\% carvone 
(a)<smiles>C=CC(C)(O)CCC=C(C)C</smiles><smiles>C=CC(C)(CCC=C(C)C)OC(C)=O</smiles>

linalool and linalyl acetate

(b)<smiles>C=C(C)C1CC=C(C)CC1</smiles><smiles>C=C(C)C1CC=C(C)C(=O)C1</smiles><smiles>C=C(C)C1CCC(C)C(=O)C1</smiles>

limonene (plane formula) carvone (plane formula) dihydrocarvone (plane formula)<smiles>C=C(C)[C@@H]1CCC(C)[C@H](O)C1</smiles>

cis-dihydrocarveol<smiles>C=C(C)[C@@H]1CCC(C)[C@H](O)C1</smiles>

trans-dihydrocarveol<smiles>C=C(C)[C@@H]1CC=C(C)[C@H](OC(C)=O)C1</smiles>

cis-carvyl acetate<smiles>C=C(C)[C@@H]1CC=C(C)[C@H](OC(C)=O)C1</smiles>

trans-carvyl acetate

Fig. 1 a Open-chain monoterpenes, b limonene and 2-oxo-derivatives detected in M. longifolia EOs

was reported from Iran, former Yugoslavia, France, Estonia and Tajikistan (Sharopov et al. 2012; Kapp 2015).

\section{Limonene 3-oxo derivatives}

Piperitone, the two piperitone oxide isomers, piperitenone and piperitenone epoxide are typical in the EO (Başer et al. 1999; Aksit et al. 2013). Pulegone appears frequently, too. This volatile, a major component also of the pennyroyal (M. pulegium), bears an unpleasant aroma and is considered to be toxic. Target human organs are suggested to be the liver and kidney which may be damaged via reactive metabolites in the case of long-term consumption (EPA/HMPC/138386/2005 Rev 1) (European Medicines Agency, Committee on Herbal Medicinal Products (HMPC) 2016). The EU directive EC1334/2008 (EEC 2008) declares that pulegone and menthofurane are limited to max. $20 \mathrm{mg} / \mathrm{kg}$ in general foodstuff, $200 \mathrm{mg} / \mathrm{kg}$ in mint/ 
<smiles>CC(C)=C1CCC(C)CC1=O</smiles><smiles>CC1=CC(=O)C(=C(C)C)CC1</smiles>

pulegone<smiles>CC1=CC(=O)C(C(C)C)CC1</smiles>

piperitone (a)<smiles>CC1CCC(C(C)C)C(=O)C1</smiles><smiles>CC1CC[C@H](C(C)C)C(=O)C1</smiles><smiles>CC(C)[C@H]1CC[C@@](C)(O)[C@H](Cl)C1=O</smiles>

menthone<smiles>CC(C)=C1CCC2(C)OC2C1=O</smiles>

piperitenone oxide<smiles>CC(C)[C@H]1CC[C@@]2(C)O[C@H]2C1=O</smiles>

cis-piperitone oxide<smiles>CC(C)[C@H]1CCC2(C)O[C@H]2C1=O</smiles><smiles>Cc1coc2c1CCC(C)C2</smiles>

trans-piperitone oxide menthofurane

(b)<smiles>CC(C)[C@]12CC[C@@](C)(O)[C@H]1C2</smiles>

trans-sabinene hydrate<smiles>CC1=CCC(O)(C(C)C)CC1</smiles>

terpinen-4-ol<smiles>CC1=CCC(C(C)(C)O)CC1</smiles>

alpha-terpineol<smiles>CC(=O)OC(C)(C)C1CC=C(C)CC1</smiles>

alpha-terpinyl acetate<smiles>CC(C)(C)C1CCC2(C)CC2O1</smiles>

eucalyptol

(c)<smiles>C=C1CC/C=C(\C)CCC2C1CC2(C)C</smiles>

beta-caryophyllene<smiles>C=C1CCC2OC2(C)CCC2C1CC2(C)C</smiles>

beta-caryophyllene oxide<smiles>C=C(C)CCC1CCC(C)=CCCC(=C)CC1C</smiles>

germacrene D

Fig. 2 a Limonene-3-oxo-derivatives b miscellaneous cyclic monoterpenes, c sesquiterpenes detected in M. longifolia EOs 
peppermint flavoured confectionery, and $100 \mathrm{mg} / \mathrm{kg}$ in chewing gums. Proportion of pulegone in a ML EO varies between 20 and $85 \%$ (Fleisher and Fleisher 1991; Baser et al. 1999; Ghoulami et al. 2001; Güllüce et al. 2007; Sharopov et al. 2012; Kapp 2015). Further representants of limonene 3-oxo derivatives in $M$. longifolia are menthone, isomenthone, menthofurane (Mimica-Dukic and Bozin 2008; Kapp 2015) and an accession rich in menthol is also reported (LlorensMolina et al. 2017). Besides, Ali and co-workers (2002) report from the Pakistani sample mentioned above, a novel chlorinated limonene-3-oxo ketone. It is 1-hydroxy-2-chloromenthone or longifone.

\section{Other cyclic monoterpenes}

This group includes terpinen-4-ol, $\alpha$-terpineol, $\alpha$ terpinylacetate, eucalyptol, borneol, trans-sabinene hydrate, thymol etc., which were detected as major ingredients of ML EO in just a few cases. Alphaterpinyl acetate as a main compound in an EO was described independently from Northern Turkey (Baser et al. 1999), Jiloca basin in Spain (Llorens-Molina et al. 2015), and (Kapp 2015). In the Turkish sample the terpinyl ester was present in $42 \%$ of the EO, and in the Estonian oil in $48 \%$. The samples from Spain (18 individuals) showed somewhat lower proportion $(39 \%)$ of the ester. From Serbia, a unique EO composition was described with presence of thymol (13\%) together with its precursors $\gamma$-terpinene $(5 \%)$ and p-cymene (14\%) accompanied by eucalyptol (7\%), however without the typical limonene-derived ketones (Mimica-Dukić et al. 1993).

\section{Sesquiterpenes}

The majority of sesquiterpenes has been detected in EOs of horsemint as minor component except $\beta$ caryophyllene, caryophyllene oxide and germacrene $\mathrm{D}$ which are regularly demonstrated as major ingredients (Mimica-Dukić et al. 1993; Başer et al. 1999; Sharopov et al. 2012; Iqbal et al. 2013; LlorensMolina et al. 2015; Kapp 2015). Their proportions in the EO make up 2-10\%, however, in a Turkish sample of M. longifolia $\mathrm{L}$ ssp. typhioides var. typhioides $29 \%$ caryophyllene oxide and $12 \% \beta$-caryophyllene were determined (Başer et al. 1999). Besides, a major unknown was also detected by Başer and co-workers (2012) in samples from Marmara region (Turkey).
This compound, probably a sesquiterpene is characterized by a $\mathrm{GC}$ retention index RI $=2209$. It was present in the EO of a single $M$. longifolia $\mathrm{L}$ ssp. longifolia oil (35\% of EO) and in six M. longifolia $\mathrm{L}$ ssp. typhioides var typhioides oils (between 6-35\%).

Chemotypes of horsemint: open questions

Based on the mentioned varying main compounds of the EO, references declare the presence of different chemotypes. According to Mimica-Dukic and Bozin (2008) the wide diversity of EOs of wild-growing mints is observable in contrary to the relative stability of the composition of cultivated spices. Others authors conclude that EO composition of ML is highly variable even among the wild growing mints (Németh-Zámboriné 2015a) In spite of this, according to our knowledge, no summarizing survey or review of this partial area of the phytochemistry of M. longifolia has been published until now. Here, three larger typologies are considered. Başer and his group (1999) provided EO compositions and typology of Turkish (Aegean region) samples of two ML taxa. From $M$. longifolia ssp. longifolia (18 samples) five chemotypes were determined: 1() rich in piperitone oxides (2) linalool-rich or linalool-eucalyptol type (3) type based on carvone or carvone and $\beta$-caryophyllene (4) type rich in isomenthone (5) other compositions: one $\alpha$-terpinyl acetate based sample and another rich in terpinen-4-ol and trans-sabinene hydrate. M. longifolia ssp. typhioides var. typhioides (19 samples) have been classified into six chemotypes (1) rich in piperitone oxides (2) linalool-rich (3) carvone-rich (4) rich in trans-sabinene hydrate (5) type based on menthone or menthone/trans-piperiton-oxide (6) EOs based on trans-piperitone oxide/ $\beta$-caryophyllene or trans-piperitone oxide/ $\beta$-caryophyllene oxide. Another typology is provided by Mimica-Dukic and Bozin (2008) who distinguish nine chemotypes (signed as types I to IX) of the genus based on surveying both cultivated and wild-growing mint species. M. longifolia s.l. is present in five of these chemotypes: II, rich in linalool and/or linalyl acetate; III, based on carvone or dihydrocarvone; IV, dominated by piperitone or piperitenone; $\mathrm{V}$, piperitone oxides or piperitenone epoxide; IX, menthone, isomenthone or menthol (isomers) as main constituents. In this classification, the chemotype $\mathrm{V}$ group contains only M. longifolia and no other Mentha taxa were 
placed here. Interestingly, no thymol-para-cymene chemotype of $M$. longifolia is mentioned, although it was reported by the same authors earlier (MimicaDukić et al. 1993). Finally, Sharopov and co-authors (2012) list fourteen chemotypes of M. longifolia as the most important ones. This classification is supported by experimental data of $M$. longifolia samples collected from at least one, but usually 3-8 habitats. The mentioned chemotypes, marked by their key component are as follows: piperitenone epoxide; piperitone oxides; piperitone; isopiperitenone; piperitenone; carvone; trans-dihidrocarvone; pulegone; menthone; isomenthone; menthofurane; menthol; eucalyptol; borneol. Authors note that both EO composition and morphological traits of $M$. longifolia are highly diverse without mentioning any correlation between chemical and morphological traits.

Comparing the above mentioned three typology, carvone, piperitone, piperitone with its oxides can be established as the basis and the most widespread monoterpenes of ML chemotypes. Other studies mentioning different chemotypes of this species are scarce but recent works report new chemotypes too. A menthofurane rich accession of $M$. longifolia $\mathrm{L}$ ssp. polyadena from South-Africa is described by Viljoen et al. (2006). Three novel types in Teruel region, Spain have been explored via careful sampling of chemotaxonomically heterogeneous populations. These were a cis-sabinene hydrate/terpinen-4-ol, a $\alpha$-terpinyl acetate/carvyl acetate (Llorens-Molina et al. 2015) and an $\alpha$-terpineol acetate/8-acetoxy carvotanacetone type (Llorens-Molina et al. 2020) respectively.

\section{Antimicrobial properties of horsemint volatiles}

Beside flavour and aroma, the EO might contribute to the preservation of food products. The most comprehensive study (Güllüce et al. 2007) on M. longifolia EO rich in limonene-3-oxo compounds provide the antimicrobial activity against 15 species of molds and 14 strains of bacteria, and also against C. albicans. This data is highly valuable because most of the studies work with a far lower number of microorganisms and/or do not provide strain numbers. The tested EO contained cis-piperitone oxide (18.4\%), pulegone $(15.5 \%)$, piperitenone oxide $(14.7 \%)$, menthone $(7.9 \%)$, isomenthone $(6.6 \%)$, trans-piperitone oxide $(4.1 \%)$ and in lower (1-5\%) proportions limonene-2- oxo volatiles, accompanied by $6.6 \%$ thymol. MIC of this EO was lower or equal with the values of control antibiotics against the majority of the tested bacteria (except Streptococcus, Pseudomonas, Enterobacter and Brucella spp). Good anticandidic activity and efficacy against Fusarium spp. was also observed. Based on this finding, authors propose utilization of $M$. longifolia essential oil as a preservative.

Other constituents of the ML oils, like limonene and its 2-oxo derivatives were also demonstrated to show moderate antibacterial activity on a wide range of pathogens, including foodborne ones (e.g. E. coli, $P$. aeruginosa, Enterobacter sp.- strain numbers not provided), (Oumzil et al. 2002). The study of Aggarwal et al. (2002) demonstrated the activity of S(-)carvone, being present frequently in M. longifolia, was as effective against $K$. pneumoniae and Candida. The antimicrobial activity of limonene-3-oxo-ketones and their epoxides, together with the mint oils characterized by them are rather frequently studied in some cases due to their potential preservative properties. Studies on pulegone, piperitenone, piperitone and epoxides isolated from Mentha (Oumzil et al. 2002) or Satureja species (Tolossa et al. 2007) show that pulegone possesses strong antimicrobial activity. However, as the use of pulegone is limited, its direct utilisation in the food industry does not have much potential. The EO of the thymol-paracymene chemotype from Serbia (Mimica-Dukić et al. 1993) was observed to show considerable activity against $B$. subtilis, $S$. aureus, C. albicans and A. niger. A review of the antimicrobial activity of EO is given by Mikaili et al. (2013), with data on decreasing antibiotic resistance of food-borne bacteria together with remarkable effects against moulds, pathogen fungi and protozoas. Ehsani, Mahmoudi and co-workers (2012) demonstrated the preservative effects of $M$. longifolia EO (with main components pulegone, eucalyptol, menthofurane and isopulegone) in Iranian white-brined cow cheese. The combination of $150 \mathrm{ppm}$ EO and a probiotic bacterium (L. casei) showed a significantly better preservative effect against the dairy-borne pathogens L. monocytogenes and $S$. aureus, than any of the treatment alone. According to authors, the limiting factor of the EO concentration in the cheese may be its influence to organoleptic properties. In our opininon, the high proportion of pulegone isomers and menthofurane in the EO might also be dangerous. 


\section{Concluding remarks}

Among the phenolic compounds of M. longifolia, the AO value of rosmarinic acid has been declared frequently. Nevertheless, a part of the available data brings up the question if it is really the only or maybe the most important constituent of strong antiradical properties of the ML extracts. It was demonstrated, that the plant contains a couple of caffetannins and 55 various flavonoids, primarily flavones. Considering the relations of structure and antiradical activity, three groups of flavonoids may deserve attention, i) luteolin7-O-glycosides like lonicerin and cynaroside, ii) eriodyctiol derivatives (eriocitrin) and iii) derivatives of quercetin, among which rutin is as frequently reported from $M$. longifolia samples as cynaroside. Rutin content was found to be in significant correlation with the FRAP activity of M. longifolia (Patonay et al. 2017). Park et al. (2019) also observed strong antioxidant activity to their rutin-dominated $M$. longifolia sample. Further studies are suggested to determine if higher concentrations of quercetin derivatives are really universal characteristics to this species as it was described in some references above.

Unfortunately, well-established conclusions on the available literature data are facing difficulties arising primarily because of methodological problems. Analysis, partially on nonvolatile compounds, are often made on a single batch of questionable representativeness of M. longifolia. Bulk samples are unable to represent the real chemical variability of any population and repetability of the results is also hardly possible. Comparison of published results is aggravated through the missing definition of plant part and phenophase at collection, too. Thus, in lack of representativeness and detailed description of sampling methods, separate references are unable to confirm either the universal appearance of any components or the background of the detected differences. It may be proposed to screen flavonoids of $M$. longifolia on a wider range of samples instead of single charges. It seems to be necessary to detect also both the biotic and environmental factors which might influence accumulation of these compounds.

During optimalization of industrial uses, the effective solvent of polyphenols of $M$. longifolia should also be determined. In general, polar extraction results in high AO activity (Mikaili et al. 2013), nonpolar solvents such as hexane or DCM are not effective
(Iqbal et al. 2013). A recent study of extractability of M. longifolia, performed by our team on 36 samples, proposes to use water-ethanol with a 3:7 mixture which makes it possible to avoid the toxic methanol in food products (Patonay et al. 2019). The utilization of aqueous waste of EO hydrodistillation was also described as a potential useful way to gain polyphenolic antioxidants of Mentha spp. (Damien-Dorman et al. 2003a, b; Koşar et al. 2004; Shen et al. 2011).

On preservative efficacy of $M$. longifolia drug or nonvolatile extracts in the food matrix, no published data was found. In case of other mint species, some results are available. The drug of $M$. spicata in a dairy dessert under thermal treatment, inhibited lipid peroxidation (Bandopadhyay et al. 2008) with similar efficacy as tert-butyl-hydroquinone. In a highly different matrix, namely a whole raw fish, a $M$. arvensis ethanolic extract was able to increase shelf life by inhibiting lipid peroxidation and release of biogenic amines (Viji et al. 2015). Although these studies do not determine the constituents in the background of the preservative effect, it could be supposed, that phenolics were effective in inhibiting peroxidation based on their radical scavenging properties detailed above. These results allow us to anticipate that a standardized, deodorized M. longifolia extract rich in polyphenols may be a cheap and effective inhibitor of lipid peroxidation and coupled oxidative deteriorations in some sensible types of foodstuff, i.e. in dairy, meat or fish products.

Volatiles of M. longifolia show an extraordinarily wide variability. Because of the complexity of data and eventual contradictions, it can be established, that the chemotaxonomic investigation on $M$. longifolia needs further thoroughl study. It seems, that the geographical habitat is not closely connected to the abundance of any chemotype and the populations may be heterogenous in contrary to the primarily vegetative propagation behaviour of the plant (Llorens-Molina et al. 2015). To the contrary of some comprehensive studies, a well established definition of $M$. longifolia chemotypes is still lacking as chemical variability may have several backgrounds (Németh-Zámboriné 2015b). A more adequate knowledge on the occurrence and stability of chemotypes and those of the EO composition may encourage the utilization of the desired types, primarily those free of pulegone as being a potentially cheap source of flavorings. The rich spectrum of volatiles also enables the selection of 
strains or clones with different aroma characters (e.g. carvone-rich: spearmint like, linalool-rich: reminiscent to lavender, etc.). Beside the phenolics, EO of $M$. longifolia might contribute to the preservation of food products, too. Evaluation and comparison of data dealing with antimicrobial activity of $M$. longifolia volatiles is, however aggravating due to the wide and varying spectrum of the investigated microbiota strains and extraction methods as well as the missing details on the enantiomers of the investigated volatiles (Oumzil et al. 2002). Chemotypes rich in piperitone, piperitenone and correspondent oxides, might have a great value as antimicrobial agents against numerous food-borne pathogens. Usage of pulegone-rich EOs should be avoided because of the toxicity of this component.

Acknowledgements Authors gratefully acknowledge the support of the Grant EFOP-3.6.1-16-2016-00001 ' 'Complex improvement of research capacities and services at Eszterhazy Karoly University' by the European Social Fund, and by the Ministry for Innovation and Technology (Hungary) within the framework of the Higher Education Institutional Excellence Program (NKFIH-1159-6/2019) in the scope of plant breeding and plant protection researches of Szent István University.

Funding Open access funding provided by Eszterhazy Karoly University.

\section{Compliance with ethical standards}

Conflict of interest Authors have no conflict of interest or competing interest to declare.

Open Access This article is licensed under a Creative Commons Attribution 4.0 International License, which permits use, sharing, adaptation, distribution and reproduction in any medium or format, as long as you give appropriate credit to the original author(s) and the source, provide a link to the Creative Commons licence, and indicate if changes were made. The images or other third party material in this article are included in the article's Creative Commons licence, unless indicated otherwise in a credit line to the material. If material is not included in the article's Creative Commons licence and your intended use is not permitted by statutory regulation or exceeds the permitted use, you will need to obtain permission directly from the copyright holder. To view a copy of this licence, visit http://creativecommons.org/licenses/by/4.0/.

\section{References}

Aggarwal KK, Khanuja SPS, Ahmad A, Santha Kumar TR, Gupta Vivek K, Kumar S (2002) Antimicrobial activity profiles of the two enantiomers of limonene and carvone isolated from the oils of Mentha spicata and Anethum sawa. Flav Fragr J 19:59-63. https://doi.org/10.1002/ffj. 1040

Akroum S, Bendjeddou D, Satta D, Lalaoui K (2009) Antibacterial activity and acute toxicity effect of flavonoids extracted from Mentha longifolia. Am Eurasian J Sustain Agric 4:93-96

Aksit H, Demirtas I, Telci I, Tarımcilar G (2013) Chemical diversity in essential oil composition of Mentha longifolia (L.) Hudson subsp. typhoides (Briq.) Harley var. typhoides from Turkey. J Essent Oil Res 25:430-437. https://doi.org/ 10.1080/10412905.2013.829005

Ali MS, Saleem M, Ahmad W, Parvez M, Yarudani R (2002) A chlorinated monoterpene ketone, acylated glycosides of beta-sitosterol and a flavonoid glycoside of Mentha longifolia. Phytochemistry 59:889-895. https://doi.org/10. 1016/S0031-9422(01)00490-3

Al-Okbi YS, Fadel HHM, Mohamed DA (2015) Phytochemical constituents, antioxidant and anticancer activity of Mentha citrata and Mentha longifolia. Res J Pharm Biol Chem Sci 6:739-751

Areias FM, Valentao P, Andrade PM, Ferreres F, Seabra RM (2001) Phenolic fingerprint of peppermint leaves. Food Chem 73:307-311. https://doi.org/10.1016/S03088146(00)00302-2

Bahadori BM, Zengin G, Bahadori S, Dinparast L (2018) Phenolic composition and functional properties of wild mint (Mentha longifolia var. calliantha (Stapf) Briq.). Int J Food Prop 21:183-193. https://doi.org/10.1080/10942912. 2018.1440238

Bandyopadhyay M, Chakraborty R, Raychaudhury U (2008) Antioxidant activity of natural plant sources in dairy dessert (Sandesh) under thermal treatment. LWT-Food Sci Technol 41:816-825. https://doi.org/10.1016/j.lwt.2007. 06.001

Baris O, Karaday M, Yanmis M, Güvenalp Z, Bal T, Güllüce M (2011) Isolation of 3 flavonoids from Mentha longifolia (L) Hudson subsp. longifolia and determination of their genotoxic potentials by using the E coli WP2 test system. J Food Sci 79:T212-T217. https://doi.org/10.1111/j.17503841.2011.02405.x

Başer KHC, Kürkçüoğlu M, Tarimcilar G, Kaynak G (1999) Essential oils of Mentha species from Northern Turkey. J Essent Oil Res 11:579-588. https://doi.org/10.1080/ 10412905.1999.9701218

Başer KHC, Kürkçüoğlu M, Demirci B, Özek T, Tarimcilar G (2012) Essential oils of Mentha species from Marmara region of Turkey. J Essent Oil Res 24:265-272. https://doi. org/10.1080/10412905.2012.676775

Basile A, Giordano S, López-Sáez JA, Cobianchi RC (1999) Antibacterial activity of pure flavonoids isolated from mosses. Phytochemistry 52:1479-1482. https://doi.org/10. 1016/S0031-9422(99)00286-1

Benedec D, Vlase L, Oniga I, Mot AC, Silaghi-Dumitrescu R, Hanganu D, Tiperciuc BT, Crisan G (2013) LC-MS analysis and AO activity of phenolic compounds from two indigenous species of Mentha. Note I. Farmacia 61:262-267

Bertoli A, Leonardi M, Krzyzanowska J, Ołeszek W, Pistelli L (2011) Mentha longifolia in vitro cultures as safe source of 
flavouring ingredients. Acta Biochim Pol 58:581-587. https://doi.org/10.18388/abp.2011_2228

Bors W, Heller W, Michel C, Saran M (1990) Flavonoids as antioxidants: determination of radical-scavenging efficiencies. Methods Enzymol 186:343-355. https://doi.org/ 10.1016/0076-6879(90)86128-I

Bourwieg D, Pohl R (1973) The flavonoids of Mentha longifolia. Planta Med 24:304-314. https://doi.org/10.1055/s0028-1099503

Burda S, Ołeszek W (2001) Antioxidant and antiradical activity of flavonoids. J Agric Food Chem 49:2774-2779. https:// doi.org/10.1021/jf001413m

Csepregi K, Neugart S, Schreiner M, Hideg É (2016) Comparative evaluation of total antioxidant capacities of plant polyphenols. Molecules 21:208. https://doi.org/10.3390/ molecules 21020208

Damien-Dorman HJ, Koşar M, Kahlos K, Holm Y, Hiltunen R (2003) Antioxidant properties and composition of aqueous extracts from Mentha species, hybrids, varieties, and cultivars. J Agric Food Chem 51:4563-4569. https://doi.org/ 10.1021/jf034108k

Dudai N, Segev D, Havkin-Frenkel D, Eshel A (2006) Genetic variation of phenolic compounds content, essential oil composition and antioxidative activity in Israel-grown Mentha longifolia L. Acta Hortic 709:69-78. https://doi. org/10.17660/ActaHortic.2006.709.8

Ehsani A, Mahmoudi R (2012) Effects of Mentha longifolia L. essential oil and Lactobacillus casei on the organoleptic properties and on the growth of Staphylococcus aureus and Listeria monocytogenes during manufacturing, ripening and storage of Iranian white-brined cheese. Int J Dairy Technol 66:70-76. https://doi.org/10.1111/j.1471-0307. 2012.00865.x

Elansary HO, Mahmoud EA (2015) Egyptian herbal tea infusions' antioxidants and their antiproliferative and cytotoxic activities against cancer cells. Nat Prod Res 29:474-479. https://doi.org/10.1080/14786419.2014.951354c

Ertaş A, Gören AC, Hasimi N, Tolan V, Kolak U (2015) Evaluation of antioxidant, cholinesterase inhibitory and antimicrobial properties of Mentha longifolia ssp. noëana and its secondary metabolites. Rec Nat Prod 9:105-115

European Medicines Agency, Committee on Herbal Medicinal Products (HMPC) (2016) Public statement on the use of herbal medicinal products containing pulegone and menthofuran. EPA/HMPC/138386/2005 Rev 1

Farzaei MH, Bahramsoltani R, Ghobadi A, Najafi F (2017) Pharmacological activity of Mentha longifolia and its phytoconstituents. J Tradit Chin Med (JTCM) 37:710-720. https://doi.org/10.1016/S0254-6272(17)30327-8

Fialová S, Tekel'ová D, Mrlianová M, Grančai D (2008) The determination of phenolic compounds and antioxidant activity of mints and balms cultivated in Slovakia. Acta Fac Pharm Univ Comen LIV:96-102

Fleisher A, Fleisher Z (1991) Aromatic plants of the Holy Land and the Sinai. Part IV: the essential oils from Mentha longifolia growing in Sinai and Israel. J Essent Oil Res 3:57-58. https://doi.org/10.1080/10412905.1991.9697909

Freeman BL, Eggett DL, Parker TL (2010) Synergistic and antagonistic interactions of phenolic compounds found in navel oranges. J Food Sci 75:570-575. https://doi.org/10. 1111/j.1750-3841.2010.01717.x
Ghoulami S, Il-Idrissi A, Fkih-Tetouani S (2001) Phytochemical study of Mentha longifolia of Morocco. Fitoterapia 72:596-598. https://doi.org/10.1016/j.foodchem.2006.10. 061

Grayer RJ, Eckert MR, Veitch NC, Kite GC, Marin PD, Kokubun T, Simmonds MSJ, Paton AJ (2003) The chemotaxonomic significance of two bioactive caffeic acid esters, nepetoidins $\mathrm{A}$ and $\mathrm{B}$, in the Lamiaceae. Phytochemistry 64:519-528. https://doi.org/10.1016/s00319422(03)00192-4

Guédon DJ, Pasquier BP (1994) Analysis and distribution of flavonoid glycosides and rosmarinic acid in 40 Mentha $x$ piperita clones. J Agric Food Chem 42:679-684. https:// doi.org/10.1021/jf00039a015

Güllüce M, Sahin F, Sokmen M, Ozer H, Daferera D, Sokmen A, Polissiou M, Adiguzel A, Ozkan H (2007) Antimicrobial and antioxidant properties of essential oils and methanolic extract from Mentha longifolia ssp. longifolia. Food Chem 103:1449-1456. https://doi.org/10.1016/j. foodchem.2006.10.061

Hajimehdipoor H, Shahrestani R, Shekarchi M (2014) Investigating the synergistic antioxidant effects of some flavonoid and phenolic compounds. Res J Pharmacogn 1:35-40

Hajlaoui H, Trabelsi N, Noumi E, Snoussi M, Fallah H, Ksouri R, Bakhrouf A (2009) Biological activities of the essential oils and methanol extract of two cultivated mint species (Mentha longifolia and Mentha pulegium) used in the Tunisian folkloric medicine. World J Microbiol Biotechnol 25:2227-2238. https://doi.org/10.1007/s11274-009-01303

Hawrył MA, Niemiec M, Słomka K, Waksmundzka-Hajnos M, Szimczak G (2016) Micro-2D-TLC Separation of phenolics in some species of mint and their fingerprints on diol bonded polar stationary phase. Acta Chrom 28:119-127. https://doi.org/10.1556/AChrom.28.2016.1.9

Iqbal T, Hussain AI., Shahid Chatha SA, Naqvi SAR, Hussain Bokhari T (2013) Antioxidant activity and volatile and phenolic profiles of essential oil and different extracts of wild mint (Mentha longifolia) from the Pakistani flora. J Anal Meth Chem 2013, Article ID 536490. https://doi. org/10.1155/2013/536490

Jahan N, Malik A, Muhammad P (2001) New flavonoid from Mentha longifolia. Heterocycles 55:1951-1956. https:// doi.org/10.3987/COM-01-9281

Kapp K (2015) Polyphenolic and essential oil composition of Mentha and their antimicrobial effect. Dissertation, University of Helsinki

Karousou R, Lanaras T, Kokkini S (1998) Piperitone oxide-rich essential oils from Mentha longifolia ssp. petiolata and $M$. villoso-nervata grown wild on the island of Crete (S. Greece). J Essent Oil Res 10:375-379. https://doi.org/10. 1080/10412905.1998.9700926

Koşar M, Damien Dorman HJ, Başer KHC, Hiltunen R (2004) Screening of free radical scavenging compounds in water extracts of Mentha samples using a postcolumn derivatization method. J Agric Food Chem 52:5004-5010. https:// doi.org/10.1021/jf0496189

Krzyzanowska J, Janda B, Pecio L, Stochmal A, Oleszek W (2011) Determination of polyphenols in Mentha longifolia and $M$. piperita field-grown and in vitro plant samples using UPLC-TQ-MS. J AOAC Int 94:43-50 
Llorens-Molina JA, García-Rellán D, Vacas S, Bonet A (2015) Individual sampling approach to study the chemodiversity of volatile and semivolatile compounds of Mentha longifolia L. growing wild in Jiloca basin, Spain. Int J Biosci 7:166-177. https://doi.org/10.12692/ijb/7.4.166-176

Llorens-Molina JA, Castell V, Vacas S, Verdeguer M (2017) TLC-GC/MS Method for identifying and selecting valuable essential oil chemotypes from wild populations of Mentha longifolia L. NVEO 4:49-61

Llorens-Molina JA, Vacas S, Castell V, Verdeguer M (2020) Seasonal variations of essential oils from five accessions of Mentha longifolia (L.) L. with selected chemical profiles. JEOR. https://doi.org/10.1080/10412905.2020.1773328

Mahmoudi R, Tajik H, Ehsani A, Farshid A, Zare P, Hadian M (2012) Effects of Mentha longifolia L. essential oil on viability and cellular ultrastructure of Lactobacillus casei during ripening of probiotic Feta cheese. Int J Dairy Technol 66:77-82. https://doi.org/10.1111/j.1471-0307. 2012.00867.x

Metsämuuronen S, Sirén H (2019) Bioactive phenolic compounds, metabolism and properties: a review on valuable chemical compounds in Scots pine and Norway spruce. Phytochem Rev 18:623-664. https://doi.org/10.1007/ s11101-019-09630-2

Mikaili P, Mojaverrostami S, Milad Moloudizargari M, Aghajanshakeri S (2013) Pharmacological and therapeutic effects of Mentha Longifolia L. and its main constituent, menthol. Anc Sci Life 33:131-138. https://doi.org/10. 4103/0257-7941.139059

Mimica-Dukić N, Bozin B (2008) Mentha L species (Lamiaceae) as promising sources of bioactive secondary metabolites. Curr Pharm Des 14:3141-3150. https://doi. org/10.2174/138161208786404245

Mimica-Dukić N, Kite G, Gasic O, Stajner D, Pavkov R, Jancic R, Fellows L (1993) Comparative study of volatile constituents and antimicrobial activity of Mentha species. Acta Hortic 344:110-115. https://doi.org/10.17660/ActaHortic. 1993.344.12

Mimica-Dukić N, Jakovljevic V, Popovic M, Gašic O, Szabo A (1996) Pharmacological study of Mentha longifolia phenolic extracts. Int J Pharmacogn 34:359-364. https://doi. org/10.1076/phbi.34.5.359.13253

Mimica-Dukić N, Jakovljevic V, Popovic M, Gašic O, Szabo A (1999) Pharmacological study of Mentha longifolia phenolic extracts II. Hepatoprotective activity. Pharm Biol 37:221-224. https://doi.org/10.1076/phbi.37.3.221.6306

Murad HAS, Abdallah HM, Ali SS (2016) Mentha longifolia protects against acetic-acid induced colitis in rats. J Ethnopharm 190:354-361. https://doi.org/10.1016/j.jep. 2016.06.016

Németh-Zámboriné É (2015a) Natural variability of essential oil components. In: Başer KHC, Buchbauer G (eds) Handbook of essential oils, 2nd edn. CRC Press - Taylor and Francis Group LLC, Boca Raton, p 95

Németh-Zámboriné É (2015b) Natural variability of essential oil components. In: Başer KHC, Buchbauer G (eds) Handbook of essential oils, 2nd edn. CRC Press - Taylor and Francis Group LLC, Boca Raton, p 116

Olszowy-Tomczyk M (2020) Synergistic, antagonistic and additive antioxidant effects in the binary mixtures. Phytochem Rev. https://doi.org/10.1007/s11101-019-09658-4
Orav A, Kapp K, Raal A (2013) Chemosystematic markers for the essential oils in leaves of Mentha species cultivated or growing naturally in Estonia. P Est Acad Sci 62:175-186. https://doi.org/10.3176/proc.2013.3.03

Orhan F, Barıs Ö, Yanmıs D, Bal T, Güvenalp Z, Güllüce M (2012) Isolation of some luteolin derivatives from Mentha longifolia ssp. longifolia and determination of their genotoxic activities. Food Chem 135:764-769

Oumzil H, Ghoulami S, Rhajaoui M, Ilidrissi A, Fkih-Tetouani S, Faid M, Benjouad M (2002) Antibacterial and antifungal activity of essential oils of Mentha suaveolens. Phytoter Res 16:727-731. https://doi.org/10.1002/ptr.1045

Park YJ, Baek SA, Choi Y, Kim JK, Park SU (2019) Metabolic profiling of nine Mentha species and prediction of their antioxidant properties using chemometrics. Molecules 24:258-273. https://doi.org/10.3390/molecules24020258

Patonay K, Korózs M, Murányi Z, Pénzesné Kónya E (2017) Polyphenols in northern Hungarian Mentha longifolia (L.) L. treated with ultrasonic extraction for potential oenological uses. Turk J Agric For 41:208-217. https://doi.org/ 10.3906/tar-1701-61

Patonay K, Szalontai H, Csugány J, Szabó-Hudák O, Helga Pénzesné Kónya E, Zámboriné Németh É (2019) Comparison of extraction methods for the assessment of total polyphenol content and in vitro antioxidant capacity of horsemint (Mentha longifolia (L.) L.). J Appl Res Med Aromat Plants 15:100220. https://doi.org/10.1016/j. jarmap.2019.100220

Pereira OR, Cardoso SM (2013) Overview of Mentha and Thymus polyphenols. Curr Anal Chem 9:382-396. https:// doi.org/10.2174/1573411011309030008

Petersen M, Simmonds MSJ (2003) Molecules of interestrosmarinic acid. Phytochemistry 62:121-125. https://doi. org/10.1016/S0031-9422(02)00513-7

Reber JD, Eggett DL, Parker TL (2011) Antioxidant capacity interactions and a chemical/structural model of phenolic compounds found in strawberries. Int J Food Sci Nutr 62:445-452. https://doi.org/10.3109/09637486.2010. 549115

Regulation (EC) No 1334/2008 of the European Parliament and of the Council of 16 December 2008 on flavourings and certain food ingredients with flavouring properties for use in and on foods and amending Council Regulation (EEC) No 1601/91, Regulations (EC) No 2232/96 and (EC) No 110/2008 and Directive 2000/13/EC. Official Journal of the European Union L 354:34-50

Rice-Evans CA, Miller NJ, Paganga G (1996) Structure-activity relationships of flavonoids and phenolic acids. Free Rad Biol Med 20:933-956. https://doi.org/10.1016/08915849(95)02227-9

Sevindik M (2018) Pharmacological properties of Mentha species. J Tradit Med Clin Natur 7:259. https://doi.org/10. 4172/2573-4555.1000259

Sevindik M, Akgul H, Pehlivan M, Selamoglu Z (2017) Determination of therapeutic potential of Mentha longifolia ssp. longifolia. Fresen Environ Bull 26:4757-4763

Shahidi F, Ambigaipalan P (2015) Phenolics and polyphenolics in foods, beverages and spices: antioxidant activity and health effects-a review. J of Funct Foods 18:820-897. https://doi.org/10.1016/j.jff.2015.06.018 
Sharaf M, El-Ansari M, Saleh NAM (1999) Flavonoid glycosides from Mentha longifolia. Fitoterapia 70:478-483. https://doi.org/10.1016/S0367-326X(99)00062-3

Sharopov FS, Sulaimanova VA, Setzer WN (2012) Essential oil composition of Mentha longifolia from wild populations growing in Tajikistan. J. Med Active Plants 1:76-84. https://doi.org/10.7275/R5736NTN

Shekarchi M, Hajimehdipoor H, Saeidnia S, Gohari RA (2012) Comparative study of rosmarinic acid content in some plants of Labiatae family. Phcog Mag 8:37-41. https://doi. org/10.7275/R5736NTN

Shen D, Pan HM, Wu QL, Park CH, Rodolfo Juliani H, Ho CT, Simon JE (2011) A rapid LC/MS/MS method for the analysis of nonvolatile antiinflammatory agents from Mentha spp. J Food Sci 76:C900-C908. https://doi.org/10. 1111/j.1750-3841.2011.02281.x

Stanislavljević DM, Stojičević SS, Đorđević SM, Zlatković BP, Veličković DT, Karabegović IT (2012) Antioxidant activity, the content of total phenols and flavonoids in the ethanol extracts of Mentha longifolia $\mathrm{L}$. dried by the use of different techniques. Chem Ind Chem Eng Q 18:411-420. https://doi.org/10.2298/CICEQ110919017S

Stocker M, Pohl R (1976) Postmortale bildung von 5,7-dihydroxychromon-7-rutinosid in Mentha longifolia. Phytochemistry 15:571-572. https://doi.org/10.1016/S00319422(00)88984-0

Tahira R, Naeemullah M, Akbar F, Masood MS (2011) Major phenolic acids of local and exotic mint germplasm grown in Islamabad. Pak J Bot 43:151-154

Tolossa K, Asres K, El-Fiky FK, Singab ANB, Bucar F (2007) Composition of the essential oils of Satureja abyssinica ssp. abyssinica and Satureja paradoxa: their antimicrobial and radical scavenging activities. J Essent Oil Res 19:295-300. https://doi.org/10.1080/10412905.2007. 9699285

Tomás-Barberán FA, Husain SZ, Gil MI (1988) The distribution of methylated flavonoids in the Lamiaceae. Biochem Syst Ecol 16:43-46. https://doi.org/10.1016/03051978(88)90115-9

Tucker AO, Naczi RFC (2007) Mentha: an overview of its classification and relationships. In: Lawrence BM (ed) Mint. The genus Mentha - Medicinal and aromatic plants - Industrial profiles. CRC Press, Boca Raton
Tunçtürk M, Tunçtürk R, Sekeroglu N, Ertus MM, Ozgokce F (2011) Lead concentrations of herbs used in Van Herby cheese. Nat Prod Commun 6:1473-1474

Ulubelen A, Topcu U, Kolak U (2005) Labiatae flavonoids and their bioactivity. Stud Nat Prod Chem 30:233-302. https:// doi.org/10.1016/S1572-5995(05)80035-3

Venskutonis PR (1996) A chemotype of Mentha longifolia L. from Lithuania rich in piperitenone oxide. J Ess Oil Res 8:91-95. https://doi.org/10.1080/10412905.1996.9700564

Viji P, Binsi PK, Visnuvinayagam S, Bindu J, Ravishankar CN, Gopal TKS (2015) Efficacy of mint (Mentha arvensis) leaf and citrus (Citrus aurantium) peel extracts as natural preservatives for shelf life extension of chill stored Indian mackerel. J Food Sci Technol. https://doi.org/10.1007/ s13197-015-1788-1

Viljoen M, Petkar S, van Vuuren SF, Figueiredo AC, Pedro LG, Barroso JG (2006) The chemo-geographical variation in EO composition and the antimicrobial properties of "Wild Mint"-Mentha longifolia subsp. polyadena. J Essent Oil Res 18:60-65. https://doi.org/10.1080/10412905.2006. 12067123

Vladimir-Knežević S, Blažeković B, Kindl M, Vladić J, LowerNedza AD, Brantner AH (2014) Acetylcholinesterase inhibitory, antioxidant and phytochemical properties of selected medicinal plants of the Lamiaceae family. Molecules 19:767-782. https://doi.org/10.3390/ molecules 19010767

Wen L, Wu D, Jiang Y, Nagendra PK, Lin S, Jiang G, Zhao M, Luo W, Yang B (2014) Identification of flavonoids of litchi (Litchi chinensis Sonn.) leaf and evaluation of anticancer activities. J Funct Foods 6:555-563. https://doi.org/10. 1016/jff.2013.11.022

Younis MHY, Beshir SM (2011) Carvone-rich essential oils from Mentha longifolia (L.) Huds. ssp. schimperi Briq. and Mentha spicata L. grown in Sudan. J Ess Oil Res 16:539-541. https://doi.org/10.1080/10412905.2004. 9698792

Publisher's Note Springer Nature remains neutral with regard to jurisdictional claims in published maps and institutional affiliations. 